\title{
Leggett modes in iron-based superconductors as a probe of time-reversal symmetry breaking
}

\author{
M. Marciani, ${ }^{1}$ L. Fanfarillo, ${ }^{2,1}$ C. Castellani, ${ }^{1}$ and L. Benfatto ${ }^{1}$ \\ ${ }^{1}$ Institute for Complex Systems (ISC), CNR, U.O.S. Sapienza and Department of Physics, Sapienza University of Rome, \\ P.le A. Moro 2, 00185 Rome, Italy \\ ${ }^{2}$ Instituto de Ciencia de Materiales de Madrid, ICMM-CSIC, Cantoblanco, E-28049 Madrid, Spain
}

(Received 27 June 2013; published 10 December 2013)

\begin{abstract}
Since their discovery, it has been suggested that pairing in pnictides can be mediated by spin fluctuations between hole and electron bands. In this view, multiband superconductivity would substantially differ from other systems like $\mathrm{MgB}_{2}$, where pairing is predominantly intraband. Indeed, interband-dominated pairing leads to the coexistence of bonding and antibonding superconducting channels. Here, we show that this has profound consequences on the nature of the low-energy superconducting collective modes. In particular, the so-called Leggett mode for phase fluctuations is absent in the usual two-band description of pnictides. On the other hand, when also the repulsion between the hole bands is taken into account, a more general three-band description should be used, and a Leggett mode is then allowed. Such a model, which has been proposed for strongly hole-doped 122 compounds, can also admit a low-temperature $s+i s$ phase that breaks the time-reversal symmetry. We show that the (quantum and thermal) transition from the ordinary superconductor to the $s+i s$ state is accompanied by the vanishing of the mass of Leggett-like phase fluctuations, regardless the specific values of the interaction parameters. This general result can be obtained by means of a generalized construction of the effective action for the collective degrees of freedom that allows us also to deal with the nontrivial case of dominant interband pairing.
\end{abstract}

DOI: 10.1103/PhysRevB.88.214508

PACS number(s): 74.20.-z, 74.70.Xa, 74.40.-n

\section{INTRODUCTION}

At microscopic level, the appearance of superconductivity requires the pairing of electrons into Cooper pairs, which can then form a macroscopic coherent state responsible for the superfluid behavior. Within BCS theory, which successfully explained the superconducting (SC) phenomenon in the so-called conventional superconductors, electrons can overcome their mutual repulsion thanks to the presence of phonons, which overscreen the Coulomb repulsion leading to a residual attraction responsible for the pairing. However, such a mechanism poses an upper limit to the attainable transition temperature, which has been widely exceeded in the so-called high-temperature superconductors, such as cuprate or iron-based systems. ${ }^{1,2}$ In all these cases, superconductivity emerges and/or competes with strong electron-electron repulsion that can be accommodated by Cooper pairs by means of an unconventional form of the wave function, as it is the case in cuprates, where the $d$-wave symmetry of pairing allows the pairs to overcome the on-site Hubbard-like repulsion. In the case of pnictides, the mechanism is somehow similar, once the multiband nature of the Fermi surface is taken into account. Indeed, it has been suggested by several microscopic approaches ${ }^{3}$ that at low energy the intraband Coulomb repulsion is overcome by the interband repulsion, which allows the pairs to be formed in different bands with a gap having opposite sign, the so-called $s^{ \pm}$symmetry. Roughly speaking, such a sign change converts a repulsion in attraction, making the pair formation possible. Notice that such a pairing mechanism is fundamentally different from the one observed in other multiband superconductors, such as, e.g., $\mathrm{MgB}_{2}$. Here, indeed, the largest pairing channel is the intraband phononic one ${ }^{4}$ and the interband interaction is only responsible for a relatively small Josephson-like coupling of pairs in different bands. In this respect, pnictide superconductors represent a completely different class of SC systems with respect to $\mathrm{MgB}_{2}$.

A fundamental question associated to the unconventional nature of pairing is how it can affect the behavior of the SC collective modes, which in turn can influence the observable physical quantities, giving indirect information on the nature of the underlying SC state. Such an issue has been widely discussed in the past within the context of cuprate superconductors, ${ }^{5-11}$ and it has been the subject of intense investigation in the recent literature on pnictide superconductors. ${ }^{12-17}$ Here, the issue is made even more involved by the presence of several bands that would suggest the presence of multiple collective modes associated to the fluctuations of the amplitude and phase of the condensates in the various bands. For example, the possibility to observe the so-called Leggett mode ${ }^{18}$ that corresponds to the relative density (phase) fluctuations of the condensate in the various bands has been discussed. ${ }^{12,15}$ As it has been shown long ago in a seminal paper by Leggett, ${ }^{18}$ such a massive mode could eventually lie below the threshold for particle-hole excitations, avoiding then its overdamping. Such a situation is partly realized in $\mathrm{MgB}_{2},{ }^{20-23}$ where indeed experimental signatures of the Leggett mode have been identified in Raman spectroscopy. ${ }^{22}$ In the case of pnictides, the intriguing possibility that the Leggett mode ${ }^{14,15,17}$ becomes massless at the quantum transition between an ordinary $s^{ \pm}$state and a time-reversal-symmetry broken (TRSB) state has been also suggested. Such a TRSB state can emerge, for example, in a three-band case when interband repulsion is equally large between all the bands: ${ }^{24-27}$ in this situation, the sign change between one band and the remaining two is frustrated, leading to an intrinsically complex order parameter $\left(\Delta_{1}^{*}, \Delta_{2}^{*}, \Delta_{3}^{*}\right) \neq$ $\left(\Delta_{1}, \Delta_{2}, \Delta_{3}\right){ }^{24}$ Since the emergence of a massless collective mode could bear several observable consequences in physical 
observables, such as, e.g., Raman response ${ }^{15}$ or intervortex interactions, ${ }^{14}$ it could be used as a smoking gun to test the appearance or not of a TRSB state in pnictides.

Quite interestingly, the theoretical investigation of the properties of collective modes in pnictides suffered until now of a fundamental limitation. Indeed, as we discussed at the beginning, pairing in pnictides arises mainly from interband interactions. However, very often, a modelization has been used in the literature based on multiband models with predominant intraband pairing interactions. ${ }^{13-15}$ While this makes it possible to derive the collective modes using standard procedures based on the construction of the effective action for the collective degrees of freedom, ${ }^{13,15}$ it makes these results unsuitable for the specific case of pnictides. On the other hand, an alternative derivation based on the direct diagrammatic derivation of the collective response functions, as the one used in Refs. 16 and 17, does not allow for a simple general understanding of the number and nature of the collective modes. As we discuss in the present paper, the difference between the two cases is not only quantitative but qualitative. Indeed, when interband interaction dominates, as it is the case physically relevant for pnictides, the number itself of available low-energy collective modes is smaller than the number of bands involved in the problem. In this case, the correct understanding of the SC collective modes should be based on the number of SC bonding channels, which is usually smaller than the number of bands involved. This leads to several profound differences between pnictides and ordinary (intrabanddominated) multiband superconductors like, e.g., $\mathrm{MgB}_{2}$.

A powerful root to enlighten these differences is the explicit construction of the action for the collective modes starting from a microscopic model for pnictides that assumes predominant interband pairing. In the ordinary case of intraband-dominated pairing, such a procedure relies on the use of the so-called Hubbard-Stratonovich (HS) decoupling of the SC interaction by means of a bosonic fields associated to the pairing operators. ${ }^{28}$ This approach has been successfully applied to two ${ }^{20}$ or three-band ${ }^{13,15}$ models with predominant intraband pairing. However, when interband coupling dominates, as it is the case for pnictides, the HS decoupling must be properly modified to account for the presence of antibonding SC channels, an issue that has been often overlooked in the recent literature in the derivation of effective functionals both above e $^{17,30}$ and below ${ }^{12} T_{c}$. Here, we follow instead the strategy outlined recently in Ref. 29, where the correct implementation of the HS procedure has been used to describe the fluctuations above $T_{c}$. We then introduce a transformation of the pairing fields in the various bands that allows us to show that below $T_{c}$ the fluctuations associated to the antibonding SC channels do not give rise to observable collective modes. This result follows immediately from a general correspondence between the low-energy collective phase fluctuations and the multiband mean-field equations. When applied to the two- or three-band case with dominant interband pairing, relevant for pnictides, this correspondence allows one to show that (i) in the two-band case the Leggett mode is absent, in contrast to intraband-dominated superconductors as $\mathrm{MgB}_{2}$ and (ii) in the three-band case a Leggett mode is present, it becomes massless at the TRSB transition and it acquires again a small mass inside the TRSB phase due to the mixing to amplitude fluctuations. A second low-energy mode appears in the TRSB state, even though it is usually found very near to the threshold for single-particle excitations. In contrast to the previous literature, which focused on the softening of the Leggett mode at $T=0$ as a function of the SC coupling leading the system through a quantum phase transition to a TRSB state, ${ }^{15-17}$ we discuss its occurrence as a function of temperature. Indeed, the thermal phase transition between a TRS and TRSB phase is possibly realized in a much wider range of parameters for realistic systems, and then it has definitively more chances to be observed experimentally.

The structure of the paper is the following. In Sec. II A, we outline the main steps that lead to the effective action for the collective degrees of freedom starting from a microscopic two-band model with interband-dominated pairing. The character of the amplitude and phase modes is discussed in Sec. II B, where we also show the absence of the ordinary Leggett mode, found instead in two-band superconductors with intraband-dominated pairing. The three-band case is discussed in Sec. III. Section III A is devoted to a brief review of the possible relevance for pnictides of three-band models that admit a TRSB state. The general structure of the collective modes is discussed in Sec. III B, where it is established the correspondence between the TRSB transition and the vanishing of the mass of a Leggett-like mode. In Sec. III C, we consider a specific set of SC couplings to show explicitly the temperature (and quantum) evolution of the low-energy modes across the TRSB transition. The results of Fig. 4 summarize the main physical messages relevant for the reader who is not interested in the theoretical aspects of their derivation, and Sec. III D contains a general discussion on the experimental probes that can be used to test the behavior of the phase collective modes near the TRSB state. Section IV contains our final remarks and the summary of the main results of the paper. Additional technical details, which are useful to make a direct comparison with previous work in the literature, are reported in the appendices. Appendix A shows the equivalence between the derivation of the Gaussian action for SC fluctuations done in polar or Cartesian coordinates. Appendix B discusses the two-band case with dominant intraband pairing by means of the formalism of the present manuscript. Finally, in Appendix C, we discuss the general connection between the TRSB transition and the effective action for the three-band model.

\section{THE EFFECTIVE ACTION FOR A TWO-BAND MODEL}

\section{A. Construction of the effective action}

To show explicitly the peculiar role of interband interactions in determining the nature of the collective modes, we first describe the two-band case. Having in mind pnictide systems, such an effective modelization is usually appropriate for systems not too far away from half-filling. Indeed, in this case, one can assume that the most relevant interactions are between the two hole pockets centered at $\Gamma$ and the two electron ones centered at $M$, with no interaction between the hole bands (see also discussion in Sec. III A below). Assuming also that the electron bands are degenerate, this four-band model can be 
mapped $^{29,31}$ into an effective BCS-like two-band one as

$$
\begin{gathered}
H=H_{0}+H_{\mathrm{int}}, \quad H_{0}=\sum_{\mathbf{k}, l, \sigma} \xi_{\mathbf{k}}^{l} c_{\mathbf{k} \sigma}^{l \dagger} c_{\mathbf{k} \sigma}^{l}, \\
H_{\mathrm{int}}=-\sum_{\mathbf{q}, i j} \hat{g}_{l m} \phi_{l, \mathbf{q}}^{\dagger} \phi_{m, \mathbf{q}} \quad(l, m=1,2),
\end{gathered}
$$

where

$$
\phi_{l, \mathbf{q}}=\sum_{\mathbf{k}} c_{\mathbf{k}+q \downarrow}^{l} c_{-\mathbf{k} \uparrow}^{l}
$$

is the pairing operator in each band and the matrix $\hat{g}_{l m}$,

$$
\hat{g}=\left(\begin{array}{ll}
\alpha & \gamma \\
\gamma & \beta
\end{array}\right), \quad \operatorname{det} \hat{g}<0,
$$

describes predominant interband pairing. The bare electronic dispersion in Eq. (1) will be approximated with a parabolic one, $\xi_{\mathbf{k}}^{l}=\varepsilon_{0}^{l} \pm \mathbf{k}^{2} / 2 m_{l}-\mu$, with the plus or minus sign for electrons or holes, respectively, and the chemical potential $\mu$ will be taken equal to zero. We notice that while to account quantitatively for the correct spectral and thermodynamic properties of pnictides a more refined Eliashberg-like multiband approach is needed, ${ }^{32}$ the Hamiltonian (1) can be considered an appropriate starting point to discuss the general structure of collective modes in most pnictides.

As customary, the microscopic effective model for the collective modes can be derived by considering the action corresponding to the Hamiltonian (1), within the finitetemperature Matsubara formalism,

$$
S=\int_{0}^{\beta} d \tau\left\{\sum_{l, \mathbf{k} \sigma} c_{\mathbf{k} \sigma}^{l \dagger}(\tau)\left[\partial_{\tau}+\xi_{\mathbf{k}}\right] c_{\mathbf{k} \sigma}^{l}(\tau) d \tau+H_{I}(\tau)\right\},
$$

where $\tau$ is the imaginary time and $\beta=1 / T$. To obtain the effective action in terms of the order-parameter collective degrees of freedom, the interaction $H_{I}$ is usually decoupled in the particle-particle channel by means of the HubbardStratonovich ${ }^{33}$ field $h_{\mathrm{HS}}$ :

$$
e^{ \pm \Lambda \phi^{\dagger} \phi}=\int \mathcal{D} h_{\mathrm{HS}} e^{-\left|h_{\mathrm{HS}}\right|^{2} / \Lambda+\sqrt{ \pm 1}\left(\phi^{\dagger} h_{\mathrm{HS}}+\text { H.c. }\right)} .
$$

In the above equation, the imaginary unit $\sqrt{-1} \equiv i$ signals the presence of a repulsive particle-particle interaction. In the usual single-band case, ${ }^{28}$ one deals with an interaction attractive in the particle-particle channel, so no imaginary unit appears. However, in the present multiband case with predominant interband coupling, the diagonalization of the $\hat{g}$ matrix with a proper rotation $R$ will lead, in general, also to a negative eigenvalue, corresponding to repulsion in the particle-particle channel:

$$
\hat{g}=R^{-1} \hat{\Lambda} R=R^{-1}\left(\begin{array}{cc}
\Lambda_{1} & 0 \\
0 & -\Lambda_{2}
\end{array}\right) R, \quad \Lambda_{1,2}>0 .
$$

As we shall see below, the saddle-point values of the HS fields $h_{\mathrm{HS}}$ are connected to the SC gaps in the various bands. However, the imaginary unit in the transformation (5) would force us to shift the integration contour of $\operatorname{Re} h_{2}$ by a finite imaginary quantity, so that $\operatorname{Re}_{2} \in \mathbb{R}+i A$, see discussion below Eq. (18). To preserve an ordinary integration contour, we will enforce $A=0$ by taking advantage of the fact that the interaction Hamiltonian $H_{I}$ can be put in the diagonal form under a more general transformation $T=\hat{H}_{\varphi} R$ (with $\operatorname{det} T=1$ ), where the matrix $\hat{H}_{\varphi}$

$$
\hat{H}_{\varphi}=\left(\begin{array}{cc}
1 / \sqrt{\Lambda_{1}} & 0 \\
0 & 1 / \sqrt{\Lambda_{2}}
\end{array}\right)\left(\begin{array}{cc}
\cosh \varphi & \sinh \varphi \\
\sinh \varphi & \cosh \varphi
\end{array}\right)\left(\begin{array}{cc}
\sqrt{\Lambda_{1}} & 0 \\
0 & \sqrt{\Lambda_{2}}
\end{array}\right)
$$

leaves $\hat{\Lambda}$ invariant:

$$
\hat{H}_{\varphi}^{T} \hat{\Lambda} \hat{H}_{\varphi}=\hat{\Lambda}
$$

As one can see, the $\hat{H}_{\varphi}$ matrix is essentially proportional to the matrix of hyperbolic rotations, which commutes with the $\operatorname{diag}(1,-1)$ matrix that arises when the two eigenvalues of $\hat{g}$ have opposite sign. The relation (8) holds regardless the value of the parameter $\varphi$, which will be chosen to decouple the two SC channels, see Eq. (22) below. Indeed, thanks to Eq. (8), $\hat{g}$ can be diagonalized by $T$ as well:

$$
T=\hat{H}_{\varphi} R \Rightarrow \hat{g}=T^{T} \hat{\Lambda} T, \quad \hat{\Lambda}^{-1}=T \hat{g}^{-1} T^{T} .
$$

Thus, if we introduce the new combinations of fermionic fields

$$
\left(\begin{array}{l}
\psi_{1} \\
\psi_{2}
\end{array}\right)=T\left(\begin{array}{l}
\phi_{1} \\
\phi_{2}
\end{array}\right)
$$

$H_{\text {int }}$ can be rewritten as

$$
\begin{aligned}
H_{\mathrm{int}} & =-\sum_{\mathbf{q}, l m} \hat{g}_{l m} \phi_{l, \mathbf{q}}^{\dagger} \phi_{m, \mathbf{q}} \\
& =-\sum_{\mathbf{q}}\left(\Lambda_{1} \psi_{1, \mathbf{q}}^{\dagger} \psi_{1, \mathbf{q}}-\Lambda_{2} \psi_{2, \mathbf{q}}^{\dagger} \psi_{2, \mathbf{q}}\right)
\end{aligned}
$$

Once we define the new combinations of fermionic fields ${ }^{34}$ $\psi_{i}$, we can use the HS decoupling (5) to write the following partition function:

$$
\begin{aligned}
Z= & \int \mathcal{D} c_{\sigma}^{l} \mathcal{D} c_{\sigma}^{l^{\dagger}} \mathcal{D} h_{i} \mathcal{D} h_{i}^{\dagger} e^{-S}, \\
S= & S_{0}+\sum_{q} \frac{\left|h_{1, q}\right|^{2}}{\Lambda_{1}}+\frac{\left|h_{2, q}\right|^{2}}{\Lambda_{2}} \\
& -\sum_{q}\left(h_{1, q}^{*} \psi_{1, q}+\text { H.c. }\right)-i\left(h_{2, q}^{*} \psi_{2, q}+\text { H.c. }\right),
\end{aligned}
$$

where $q \equiv\left(i \Omega_{m}, \mathbf{q}\right)$. The action (12) is now quadratic in the fermionic fields, which can be integrated out exactly. By introducing the Nambu operators $N_{l, k}^{\dagger}=\left(c_{k, \uparrow}^{l \dagger}, c_{-k, \downarrow}^{l}\right)$, we can indeed rewrite the action as

$$
\begin{aligned}
S= & \sum_{l k, k^{\prime}} N_{l, k}^{\dagger}\left[-\bar{G}_{k, l}^{-1} \delta_{k, k^{\prime}}+\Sigma_{k, k^{\prime}}^{l}\right] N_{l, k^{\prime}} \\
& +\sum_{q} \frac{\left|h_{1, q}\right|^{2}}{\Lambda_{1}}+\frac{\left|h_{2, q}\right|^{2}}{\Lambda_{2}}
\end{aligned}
$$


where

$$
\begin{gathered}
\bar{G}_{k, l}^{-1}=\left(\begin{array}{cc}
i \omega_{n}-\xi_{k, l} & T_{1 l} \bar{h}_{1}+i T_{2 l} \bar{h}_{2} \\
T_{1 l} \bar{h}_{1}^{*}+i T_{2 l} \bar{h}_{2}^{*} & i \omega_{n}+\xi_{k, l}
\end{array}\right), \\
\Sigma_{q=k-k^{\prime}}^{l}=\sqrt{\frac{T}{V}}\left(\begin{array}{cc}
0 & T_{1 l} h_{1, q}+i T_{2 l} h_{2, q} \\
T_{1 l} h_{1, q}^{*}+i T_{2 l} h_{2, q}^{*} & 0
\end{array}\right) .
\end{gathered}
$$

In Eq. (14), we put $\bar{h}_{i}=\sqrt{T / V} h_{i, 0}$. By integrating out the fermions, one gets as usual a contribution to the action equal to $-\ln \operatorname{det}\left(\bar{G}^{-1}-\Sigma\right)=-\operatorname{Tr} \ln \left(\bar{G}^{-1}-\Sigma\right)=$ $-\operatorname{Tr} \ln \bar{G}^{-1}-\operatorname{Tr} \ln (1-\bar{G} \Sigma)$, where the trace acts both on momentum and Nambu space. One can then separate the mean-field action from the fluctuating part as

$$
\begin{gathered}
S=S_{\mathrm{MF}}+S_{\mathrm{FL}}, \\
S_{\mathrm{MF}}=\frac{\bar{h}_{1}^{2}}{\Lambda_{1}}+\frac{\bar{h}_{2}^{2}}{\Lambda_{2}}-\sum_{l} \operatorname{Tr} \ln \bar{G}_{l}^{-1}, \\
S_{\mathrm{FL}}=\sum_{q} \frac{\left|h_{1, q}\right|^{2}}{\Lambda_{1}}+\frac{\left|h_{2, q}\right|^{2}}{\Lambda_{2}}+\sum_{l} \sum_{n} \operatorname{Tr} \frac{\left(\bar{G}_{l} \Sigma\right)^{n}}{n} .
\end{gathered}
$$

From Eq. (14), one can see that the HS fields play the role of the SC gaps in each band, provided that one assumes a saddle-point value of the antibonding field such that

$$
\bar{h}_{2}=i A, \quad \bar{h}_{2}^{*}=i A,
$$

to guarantee the Hermitian form of the saddle-point action. Here, we will use instead the generalized transformation (10) to impose $\bar{h}_{2}=\bar{h}_{2}^{*}=0$ at the saddle point. This can be understood by minimizing the mean-field action (17), which gives the set of equations

$$
\left(\begin{array}{cc}
\frac{1}{\Lambda_{1}}-\sum_{l} T_{1 l}^{2} \Pi_{l} & -\sum_{l} T_{1 l} \Pi_{l} T_{2 l} \\
-\sum_{l} T_{2 l} \Pi_{l} T_{1 l} & -\frac{1}{\Lambda_{2}}-\sum_{l} T_{2 l}^{2} \Pi_{l}
\end{array}\right)\left(\begin{array}{c}
\bar{h}_{1} \\
i \bar{h}_{2}
\end{array}\right)=0,
$$

where we defined the Cooper bubble $\Pi_{l}$ as

$$
\Pi_{l}=\frac{T}{V} \sum_{\mathbf{k}, n} \frac{1}{\omega_{n}^{2}+E_{\mathbf{k}, l}^{2}},
$$

with the identification $E_{\mathbf{k}, l}^{2}=\xi_{\mathbf{k}, l}^{2}+\left[T_{1 l}^{2} \bar{h}_{1}^{*} \bar{h}_{1}-T_{2 l}^{2} \bar{h}_{2}^{*} \bar{h}_{2}+\right.$ $\left.2 i T_{1 l} T_{2 l}\left(\bar{h}_{1}^{*} \bar{h}_{2}+\bar{h}_{1} \bar{h}_{2}^{*}\right)\right]$. Once again, this quantity cannot be identified with the energy of the quasiparticles in each band, unless we use Eq. (19). On the other hand, we can choose the $\varphi$ parameter of the transformation (9) to decouple the two saddle-point equations (20):

$$
\sum_{l} T_{1 l} \Pi_{l} T_{2 l}=0 .
$$

In this case, one immediately sees that since $\Pi_{l}>0$ the equation for $\bar{h}_{2}$ can only be satisfied for $\bar{h}_{2}=0$, so that the SC transition is only controlled by the bonding field $\bar{h}_{1}$, whose self-consistent equation is

$$
\left(\frac{1}{\Lambda_{1}}-\sum_{l} T_{1 l}^{2} \Pi_{l}\right) \bar{h}_{1}=0
$$

where we also assumed that $\bar{h}_{1}$ is real. This choice corresponds to the gauge where both gaps are real, as given by [see
Eq. (14)]:

$$
\Delta_{l}=T_{1 l} \bar{h}_{1} .
$$

We stress once more that even if the saddle-point value of the antibonding HS field $h_{2}$ vanishes both gaps are in general different from zero, and their relative strength or temperature dependence is controlled by the microscopic couplings via the elements of the $T$ matrix. The possibility to describe the SC state as a function of a single order parameter reflects the fact that at $T_{c}$ only one SC channel becomes active. To make the connection with a more standard notation, we observe that the matrix $T$ in practice diagonalizes the multiband selfconsistency equation, which is usually written as

$$
\left(\hat{g}^{-1}-\hat{\Pi}\right) \vec{\Delta}=0,
$$

where $\hat{\Pi}_{i j}=\delta_{i j} \Pi_{i}$ and $\vec{\Delta}$ is a vector formed by the gaps $\Delta_{l}$ in each band. The above equation admits a nonzero solution $\vec{\Delta}$ when the determinant vanishes, i.e., when (at least) one eigenvalue is zero. By means of the relations (9) above, we see that the set of Eqs. (20) and (22) corresponds to putting the matrix $\left(\hat{\Pi}-\hat{g}^{-1}\right)$ in diagonal form,

$$
\hat{\Lambda}^{-1}-T \hat{\Pi} T^{T}=T\left(\hat{g}^{-1}-\hat{\Pi}\right) T^{T} \equiv Y_{i} \delta_{i j},
$$

so that the SC state is reached when the element $Y_{1} \equiv$ $1 / \Lambda_{1}-\sum_{l} T_{1 l}^{2} \Pi_{l}$ corresponding to the bonding eigenvalue vanishes, leading to Eq. (23) above. It should be noticed that in the two-band case, regardless the intraband or interband dominated nature of the pairing, the eigenvalues of the matrix (25) cannot be both zero, unless the interband coupling vanishes (see discussion in Appendix C). On the other hand, in the three-band case discussed in Sec. III below, the matrix (26) has three eigenvalues: as we shall see, when only one of them vanishes, one is in the usual SC phase, while the vanishing of a second eigenvalue signals the emergence of a TRSB phase. Finally, we notice also that the procedure introduced here to describe a multiband superconductor in terms of a single ordering field can be applied also to the case of spatially inhomogeneous superconducting condensates, whose Ginzburg-Landau expansion near $T_{c}$ has attracted some interest in the recent literature..$^{35,36}$

\section{B. Collective modes in the SC state}

Within the present formalism, the collective modes in the SC state can be easily obtained by expanding the action (18) up to second order in the HS fields. In the single-band case, where a single HS field is used to decouple the interaction, one can follow two alternative but equivalent roots. Indeed, as relevant variables one can use either (i) the amplitude and the phase (polar coordinates) or (ii) the real and imaginary part (Cartesian coordinates) of the HS field. In Appendix A, we show how to recover the equivalence between the two approaches. In our case, where a single HS field condenses at the transition, the second root is the only available one. On the other hand, when the interaction has a dominant intraband character, one does not need to use the transformation (10) to get rid of the antibonding field, and one can introduce HS fields associated directly to the two gaps in each band. In this case, the approach (i) can be again used, as it has been done, for example, to study the Leggett mode in $\mathrm{MgB}_{2}$ in Ref. 20, and 
more recently to investigate Leggett modes across a TRSB transition for intraband-dominating interactions in Refs. 13 and 15 . However, this is not the case physically relevant for pnictides, as we discussed in the introduction.

From Eq. (18), one can see that the coefficients of the Gaussian action for the HS field will be given in general by BCS correlation functions computed with the mean-field Green's functions (14), with the identification (24) of the band gaps. Following the straightforward procedure described in Appendix A, one then finds that

$$
\begin{gathered}
S_{\mathrm{FL}}=\sum_{q} \eta_{-q}^{T} \hat{S}_{\mathrm{FL}}(q) \eta_{q}, \\
\eta_{q}^{T}=\left(\operatorname{Re} h_{1, q}, i \operatorname{Re} h_{2, q}, \operatorname{Im} h_{1, q}, i \operatorname{Im} h_{2} q\right) .
\end{gathered}
$$

Notice that once $\bar{h}_{1}$ is fixed as real, see Eq. (24), one can identify the real and imaginary parts of the $h_{1}$ fluctuations as the leading orders in the amplitude and phase fluctuations of the field, respectively:

$$
\operatorname{Re} h_{1, q}=\left|h_{1, q}\right|, \quad \operatorname{Im} h_{1, q}=\bar{h}_{1} \theta_{1, q} .
$$

While the same identification cannot be done for the $h_{2}$ field, we can still associate its fluctuations to the real and imaginary parts of the gap fluctuations in each band. Indeed, by following the same root described in Appendix A to derive the relation between the averages of the HS fields and the averages of the physical fermionic operators (10), one can show that

$$
\begin{gathered}
\left\langle\psi_{1, q}\right\rangle=\frac{1}{\Lambda_{1}}\left\langle h_{1, q}\right\rangle, \quad\left\langle\psi_{2, q}\right\rangle=-\frac{1}{\Lambda_{2}}\left\langle i h_{2, q}\right\rangle, \\
\left\langle\psi_{1, q}^{*} \psi_{1,-q}\right\rangle=\frac{1}{\Lambda_{1}^{2}}\left(\left\langle h_{1, q}^{*} h_{1,-q}\right\rangle-\Lambda_{1}\right), \\
\left\langle\psi_{2, q}^{*} \psi_{2,-q}\right\rangle=\frac{1}{\Lambda_{2}^{2}}\left(\left\langle i h_{2, q}^{*} i h_{2,-q}\right\rangle+\Lambda_{2}\right) .
\end{gathered}
$$

Since the gap operators in each band are given by $\Delta_{l}=$ $g_{l m} \phi_{m}=T_{l m}^{T} \hat{\Lambda}_{m n} \psi_{n}$, one can also express the average values of the gap fluctuations in terms of fluctuations of the $h_{i} \mathrm{HS}$ fields as

$$
\begin{aligned}
& \left\langle\Delta_{l, q}+\Delta_{l, q}^{*}\right\rangle=T_{1 l}\left\langle\operatorname{Re} h_{1, q}\right\rangle+T_{2 l}\left\langle i \operatorname{Re} h_{2, q}\right\rangle, \\
& \left\langle\Delta_{l, q}-\Delta_{l, q}^{*}\right\rangle=T_{1 l}\left\langle\operatorname{Im} h_{1, q}\right\rangle+i T_{2 l}\left\langle i \operatorname{Im} h_{2, q}\right\rangle,
\end{aligned}
$$

and analogous expressions for the correlations functions. As a consequence, we included the imaginary unit in the $h_{2}$ components of the fluctuating vector (28) and we will refer in what follows to the first two components of $\eta_{q}$ as "amplitude" fluctuations and to the last two as "phase" fluctuations. Such a decomposition allows one also to easily identify the character of the fermionic bubbles that appear in the Gaussian action. Indeed, from Eq. (15), one sees that amplitude fluctuations are associated to a $\sigma_{1}$ Pauli matrix in the Nambu notation, while phase fluctuations to $\sigma_{2}$ [see also Eq. (A3) in Appendix A]. Moreover, as shown in Appendix A, at long-wavelength, the amplitude and phase sectors decouple, so that they are described respectively by the following $2 \times 2$ matrices:

$$
\begin{aligned}
\hat{S}_{\mathrm{FL}}^{A}(q) & =T \hat{\Lambda}^{11} T^{T} / 2+\hat{\Lambda}^{-1} \\
& =\left(\begin{array}{cc}
\frac{1}{2} \sum_{l} \Lambda_{l}^{11}(q) T_{1 l}^{2}+\frac{1}{\Lambda_{1}} & \frac{1}{2} \sum_{l} \Lambda_{l}^{11}(q) T_{1 l} T_{2 l} \\
\frac{1}{2} \sum_{l} \Lambda_{l}^{11}(q) T_{1 l} T_{2 l} & \frac{1}{2} \sum_{l} \Lambda_{l}^{11}(q) T_{2 l}^{2}-\frac{1}{\Lambda_{2}}
\end{array}\right), \\
\hat{S}_{\mathrm{FL}}^{P}(q) & =T \hat{\Lambda}^{22} T^{T} / 2+\hat{\Lambda}^{-1} \\
& =\left(\begin{array}{cc}
\frac{1}{2} \sum_{l} \Lambda_{l}^{22}(q) T_{1 l}^{2}+\frac{1}{\Lambda_{1}} & \frac{1}{2} \sum_{l} \Lambda_{l}^{22}(q) T_{1 l} T_{2 l} \\
\frac{1}{2} \sum_{l} \Lambda_{l}^{22}(q) T_{1 l} T_{2 l} & \frac{1}{2} \sum_{l} \Lambda_{l}^{22}(q) T_{2 l}^{2}-\frac{1}{\Lambda_{2}}
\end{array}\right),
\end{aligned}
$$

where the $\Lambda_{l}^{i j}$ bubbles are defined in the Appendix $\mathrm{A}$ and the corresponding diagonal matrices are $\hat{\Lambda}_{l m}^{i i} \equiv \Lambda_{l}^{i i} \delta_{l m}$. In what follows, we shall investigate the possibility that any collective mode is defined in the two sectors, by having in mind that a mode corresponds to a solution of the equation $\operatorname{det} \hat{S}_{\mathrm{FL}}(\omega=m, \mathbf{q}=0)=0$ with $m<2 \Delta_{\min }$, where $\Delta_{\text {min }}$ is the smallest gap. In practice, we are interested in well-defined resonances below the threshold of the quasiparticle excitations: thus it is enough to take into account the real part of the bubbles $\Lambda_{l}^{11}, \Lambda_{l}^{22}$ after analytical continuation $i \Omega_{m} \rightarrow \omega+i \delta$ to real frequencies, since the imaginary parts vanish at $\omega<2 \Delta_{\min }$. The different behavior of the collective modes will then follow simply from the different frequency and momentum dependence of these two bubbles, whose value at $q=0$ is connected to the Cooper bubble (21). Moreover, as it is shown in Appendix A [see Eq. (A29)], at small $q$ and low $T$, one can write

$$
\begin{gathered}
\Lambda_{l}^{11}(q)=-2 \Pi_{l}+A_{l} \Delta_{l}^{2}+\mathcal{O}\left(q^{2}\right), \\
\Lambda_{l}^{22}(q)=-2 \Pi_{l}+\frac{1}{4 \Delta_{l}^{2}}\left(\Omega_{m}^{2} \kappa_{l}+\mathbf{q}^{2} \frac{\rho_{s, l}}{m_{l}}\right),
\end{gathered}
$$

where $\kappa, \rho_{s} / m$ represent the compressibility and superfluid density of each band, respectively, and $A_{l}=$ $\sum_{\mathbf{k}} \tanh \left(\beta E_{\mathbf{k}} / 2\right) / E_{\mathbf{k}}^{3}$ [see Eq. (43) below].

By using Eq. (38), one can write down the $q=0$ limit of the phase sector (36) as

$$
\hat{S}_{\mathrm{FL}}^{P}(q=0)=\left(\begin{array}{cc}
-\sum_{l} \Pi_{l} T_{1 l}^{2}+\frac{1}{\Lambda_{1}} & 0 \\
0 & -\sum_{l} \Pi_{l} T_{2 l}^{2}-\frac{1}{\Lambda_{2}}
\end{array}\right),
$$

where we used the constraint (22) for the $T$ matrix to cancel out the off-diagonal terms at $q=0$. Equation (39) is one of the first crucial results of the use of the generalized transformation $T$; indeed, not only it decouples the saddle-point equations, but it also decouples the phase fluctuations at long wavelengths, connecting their masses to the eigenvalues of the saddle-point equations themselves, leading to a straightforward interpretation of the roles of the HS fields. Indeed, since below $T_{c} \bar{h}_{1}=0$, the self-consistent equation (23) implies that the quantity in brackets vanishes, so that one immediately sees that $\operatorname{Im} h_{1, q}$ fluctuations describe a massless mode. This is not surprising, since from Eqs. (24), (29), and (34), one sees that a phase fluctuation for the ordering $h_{1}$ field corresponds to a 
simultaneous change of the overall SC phase in all the gaps:

$$
\Delta_{l}+i T_{1 l} \operatorname{Im} h_{1}=T_{1 l} \bar{h}_{1}+i T_{1 l} \bar{h}_{1} \theta_{1} \simeq \Delta_{l} e^{i \theta_{1}} .
$$

As a consequence, $\operatorname{Im} h_{1}$ is the Goldstone mode of the SC transition, which is expected to be massless in the SC phase. For what concerns instead the fluctuations of the antibonding field $h_{2}$, we can first analyze the small frequency expansion of Eq. (36) that follows from Eq. (38), i.e.,

$$
\hat{S}_{\mathrm{FL}}^{P}=-\left(\begin{array}{cc}
\frac{\omega^{2}}{8} \sum_{l} \kappa_{l} \frac{T_{1 l}^{2}}{4 \Delta_{l}^{2}} & \frac{\omega^{2}}{8} \sum_{l} \kappa_{l} \frac{T_{1 l} T_{2 l}}{4 \Delta_{l}^{2}} \\
\frac{\omega^{2}}{8} \sum_{l} \kappa_{l} \frac{T_{1 l} T_{2 l}}{4 \Delta_{l}^{2}} & \frac{\omega^{2}}{8} \sum_{l} \kappa_{l} \frac{T_{2 l}^{2}}{4 \Delta_{l}^{2}}+\left(\sum_{l} \Pi_{l} T_{2 l}^{2}+\frac{1}{\Lambda_{2}}\right)
\end{array}\right),
$$

where the analytic continuation $i \Omega_{m} \rightarrow \omega+i \delta$ has been made. As $\omega \rightarrow 0$, one sees that $\operatorname{Im} h_{1}$ and $\operatorname{Im} h_{2}$ decouple, and one recovers the massless $\operatorname{Im} h_{1}$ mode, as discussed above. On the other hand, the fluctuations of the antibonding $h_{2}$ field do not give rise to any collective mode. Indeed, the 22 element of the matrix (41) does not admit any real solution for $\omega$, due to the fact that the quantity in brackets is strictly positive. This result, which is confirmed by the explicit calculation of $\Lambda_{l}^{22}(\omega)$ at all frequencies and temperatures, is a direct consequence of the fact that the $h_{2}$ field is associated to the antibonding SC channel of the system. Indeed, as we show in details in the Appendix B, if $h_{2}$ were associated to a bonding SC channel [i.e., a positive eigenvalue in Eq. (6)], the $-1 / \Lambda_{2}$ term in Eq. (36) would be replaced by $+1 / \Lambda_{2}$, leading to a well-defined mode in Eq. (41), which coincides with the usual Leggett mode, see Eq. (B12). It is also worth stressing that the absence of the Leggett mode in a two-band modelization of pnictides does not mean that relative phase fluctuations of the gaps in the two bands are absent, but simply that these fluctuations do not define a coherent collective mode of the system.

For what concerns instead the amplitude sector (35), by using again the self-consistent equations (22) and (23) and the relation (37), one sees that at $\mathbf{q}=0$, in general,

$$
\hat{S}_{\mathrm{FL}}^{A}=\left(\begin{array}{cc}
A+\mathcal{O}\left(\omega^{2}\right) & B+\mathcal{O}\left(\omega^{2}\right) \\
B+\mathcal{O}\left(\omega^{2}\right) & -C+\mathcal{O}\left(\omega^{2}\right)
\end{array}\right),
$$

where $A=\frac{1}{2} \sum_{l} A_{l} \Delta_{l}^{2} T_{1 l}^{2}, B=\frac{1}{2} \sum_{l} A_{l} \Delta_{l}^{2} T_{1 l} T_{2 l}$, and $C=$ $\sum_{l} C_{l} T_{2 l}^{2}+\frac{1}{\Lambda_{2}}$ are positive constants, with $C_{l}=\sum_{\mathbf{k}}\left(\xi_{\mathbf{k}}^{2} / E_{\mathbf{k}}^{3}\right)$ $\tanh \left(\beta E_{\mathbf{k}} / 2\right)$. As one could expect, there is no massless mode in the amplitude sector, since amplitude fluctuations are always costly in the SC phase. One could then wonder if massive modes are present. In the single-band case, one knows that amplitude fluctuations at $\mathbf{q}=0$ correspond to a well-defined mode with frequency $m=2 \Delta$, which get easily damped by interactions. $^{37,38}$ This result follows from the fact that the coefficient of the amplitude fluctuations reduces [see Eq. (A5)] to $(g \text { being the SC coupling })^{39}$

$$
\begin{aligned}
& \Lambda^{11}(\omega, \mathbf{q}=0)+\frac{2}{g} \\
& =\sum_{\mathbf{k}} \tanh \left(\frac{E_{\mathbf{k}}}{2 T}\right)\left[-\frac{\xi_{\mathbf{k}}^{2}}{E_{\mathbf{k}}^{2}}\left(\frac{1}{2 E_{\mathbf{k}}+\omega}+\frac{1}{2 E_{\mathbf{k}}-\omega}\right)+\frac{1}{E_{\mathbf{k}}}\right] .
\end{aligned}
$$

This function of $\omega$ vanishes at $\omega=2 \Delta$ with a square-root singularity, ${ }^{39}$ and it is positive everywhere else, see Fig. 1(a).

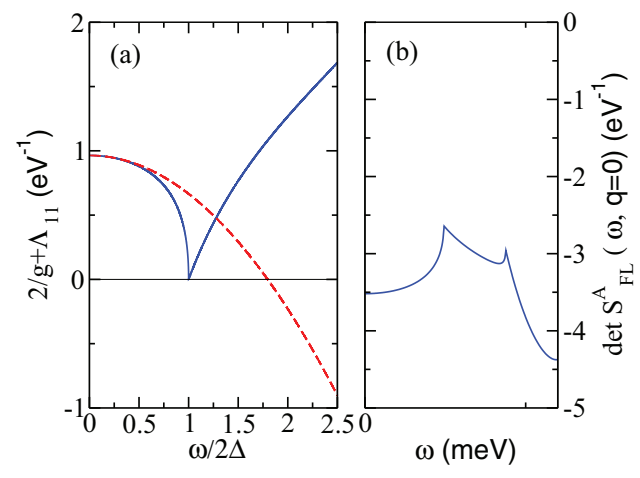

FIG. 1. (Color online) (a) Frequency dependence of the amplitude fluctuations as given by Eq. (43) in the single-band case. The vanishing at $\omega=2 \Delta$ signals the presence of an amplitude mode with mass $m=2 \Delta$. Notice that the quadratic low-frequency expansion, given by the dashed line, would lead to a wrong mass $m \simeq \sqrt{12} \Delta$. (b) Determinant of the action (35) at $T=0$ in the amplitude sector as a function of $\omega$ for coupling values $N_{1}=1 \mathrm{eV}^{-1}, N_{2}=2.2 \mathrm{eV}^{-1}$, $g_{11}=g_{22}=0, g_{12}=0.5 \mathrm{eV}$, and $\omega_{0}=15 \mathrm{meV}$. The determinant never vanishes, so that no well-defined mode is found in this case. The overall negative sign is due to the presence of the antibonding channel, see also Eq. (42).

In the multiband case described by Eq. (36) above, one is then mixing the $\Lambda_{l}^{11}$ bubbles of the two bands, which have, in general, zeros for two different values $2 \Delta_{l}$. For this reason, unless one considers strictly identical bands, the $\operatorname{det} \hat{S}_{\mathrm{FL}}^{P}(\omega, \mathbf{q}=0)$ never vanishes, as shown in Fig. 1(b), so that well-defined amplitude modes are absent. This example shows also that one should be very careful in computing the collective modes by making a low-energy expansion of the $\Lambda_{l}^{11} \simeq-2 \Pi_{l}+A_{l} \Delta^{2}-B_{l} 2 \omega^{2}$ bubbles. $^{16,17}$ Indeed, one could obtain either spurious results, or masses which are quantitatively wrong, especially in the TRSB phase where amplitude and phase fluctuations get mixed. ${ }^{14,16,17}$ We will come back to this point at the end of the next section.

Finally, we observe that above $T_{c}$ the phase and amplitude sectors become degenerate, as expected, and one recovers the results discussed in Ref. 29. Indeed, the $\Lambda_{l}^{11}$ and $\Lambda_{l}^{22}$ bubbles coincide, and the leading terms at small $q$ go like $\eta \mathbf{q}^{2}, \gamma\left|\Omega_{m}\right|$. More specifically, we observe that at $q=0$ the action for the Gaussian fluctuations coincides with the usual quadratic expansion of the free energy, and it is given by

$$
\begin{aligned}
S_{\mathrm{FL}}(q=0)= & \left(\frac{1}{\Lambda_{1}}-\sum_{l} T_{1 l}^{2} \Pi_{l}\right)\left|h_{1}\right|^{2} \\
& +\left(\frac{1}{\Lambda_{2}}+\sum_{l} T_{2 l}^{2} \Pi_{l}\right)\left|h_{2}\right|^{2} .
\end{aligned}
$$

As one can see, the coefficient of the $h_{2}$ field is always positive, showing that it never orders. In contrast, a wrong application of the HS transformation (5) lead the authors of Refs. 17 and 30 to the counterintuitive result that the coefficient of the antibonding field is always negative, making it difficult to justify why it should not order. This shows once more that an extra care is needed to extend to interband-dominated interactions the results known for single-band systems, where a single bonding SC channel exists. 

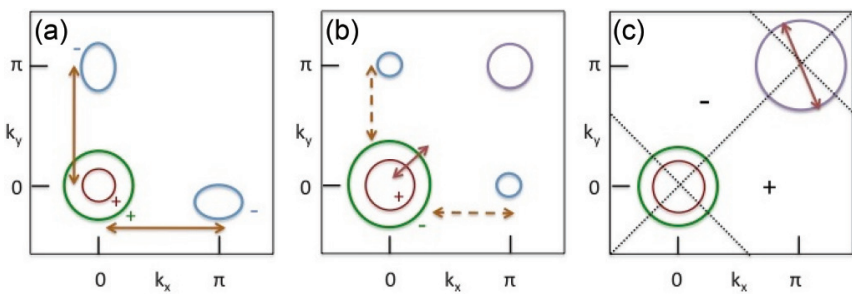

FIG. 2. (Color online) Schematic of the band structures in pnictides in the unfolded Brillouin zone. (a) Typical band structure for optimally-doped 122 compounds (like, e.g., $\mathrm{Ba}_{0.4} \mathrm{~K}_{0.6} \mathrm{Fe}_{2} \mathrm{As}_{2}$ ), formed by two hole pockets around $\Gamma$ and two electron ones at $(0, \pi)$ and $(\pi, 0)$. In this case, the largest coupling is an interband repulsion between the hole and electron Fermi sheets, leading to the $s_{ \pm}$symmetry of the order parameter with a sign change of the gap between hole and electron bands. (b) Strong hole doping: in this case, the electron pockets reduce considerably and a third hole pocket appears at $(\pi, \pi)$. In the case of $\mathrm{KFe}_{2} \mathrm{As}_{2}$ (c), the electron pockets disappear completely. It has been argued that $s$-wave (b) and $d$-wave (c) symmetries are nearby in energy at strong hole doping. In the $s$-wave symmetry, the change of sign of the gaps occurs between the two hole pockets at $\Gamma$, while in the remaining bands the order parameter is very small. In the $d$-wave symmetry (c), instead, the largest gap is on the third hole pocket and nodes are present on all the Fermi surfaces.

\section{THREE-BAND MODEL FOR THE TRSB TRANSITION}

\section{A. Occurrence of a TRSB state in pnictides}

Now that the general properties of the collective modes in a superconductor where bonding and antibonding SC channels coexist are established, let us focus more specifically on the case of a three-band model for pnictides, where an additional repulsion between the two hole bands is considered. This case has attracted considerable interest in the recent literature due to the experimental advances in making 122 samples ${ }^{42-44}$ heavily hole-doped away from half-filling, until the end member $\mathrm{KFe}_{2} \mathrm{As}_{2}$ is reached. ${ }^{44-48}$ Even though a full agreement between theoretical predictions and experimental results has not been reached yet, we would like to summarize here some results relevant for the focus of the present manuscript. A schematic of the band-structure evolution from $\mathrm{Ba}_{1-x} \mathrm{~K}_{x} \mathrm{Fe}_{2} \mathrm{As}_{2}$ to $\mathrm{KFe}_{2} \mathrm{As}_{2}$ in the unfolded Brillouin zone (one Fe atom per unit cell) is shown in Fig. 2. At intermediate doping [see Fig. 2(a)], the system admits two hole pockets at $\Gamma=(0,0)$ and two electron pockets at $(\pi, 0)$ and $(0, \pi)$. The largest interactions in this situation are the spin-fluctuation mediated interpocket repulsions between hole and electron bands, ${ }^{40,41}$ which lead to the $s_{ \pm}$symmetry of the order parameter, i.e., constant gaps on all the FS with a change of sign between hole and electron bands. In this situation, by neglecting nematic effects making the electron pockets inequivalent, an effective two-band description as the one discussed in the previous section is possible. ${ }^{29,31}$ As doping increases, the electron pockets shrink and a third hole pocket around $(\pi, \pi)$ appears [see Fig. 2(b)], until only hole pockets remain for $\mathrm{KFe}_{2} \mathrm{As}_{2}$ [see Fig. 2(c)]. In this compound, several theoretical calculations ${ }^{25,26,41}$ have shown that $s$-wave and $d$-wave symmetries are almost degenerate in energy. However, the gap hierarchy would be very different in the two cases: in the $s$-wave case, the leading interaction is an interband repulsion at small momentum between the hole pockets at $\Gamma$, so that the sign change between the gaps is now realized between the two central hole bands (having eventually accidental nodes ${ }^{27}$ ), while on the remaining pockets the gap is vanishing. Instead, the $d$-wave symmetry is driven by a large intraband repulsion within the hole pocket at $(\pi, \pi)$, so that the gap is largest here and nodes are present on all the FS. The experimental situation is quite controversial: while ARPES measurements show no nodes at large $(x=0.7) \mathrm{K}$ doping ${ }^{42,43}$ or accidental nodes for $\mathrm{KFe}_{2} \mathrm{As}_{2},{ }^{48}$ thermal probes of the quasiparticle excitations indicate nodal gaps. ${ }^{45,46}$

From the point of view of the general description of the collective modes that we will give here, the relevant aspect is that once two SC channels are almost degenerate in energy, one can eventually access a phase where both of them coexist, leading to a TRSB state. To slightly simplify the notation and to make contact with previous work on this topic, ${ }^{16,17,24}$ we will discuss here the case where the order parameter remains in the $s$-wave symmetry class, so that the most relevant interactions are interband repulsion between hole and electron bands, and within the hole pockets at $\Gamma$. By assuming again degenerate electron pockets, one can then investigate, for example, the minimal three-band model proposed in Ref. 24 where the two hole bands (bands 1,2) are equal, so that the matrix $\hat{g}$ of Eq. (3) becomes for this three-band case:

$$
\hat{g}=-\left(\begin{array}{ccc}
0 & V_{h h} & V_{h e} \\
V_{h h} & 0 & V_{h e} \\
V_{h e} & V_{h e} & 0
\end{array}\right) .
$$

As it has been noticed in Ref. 24, despite the fact that the mean-field equations in this three-band model appear as a straightforward generalization of the two-band case discussed in the previous section, the intrinsic frustration hidden in the SC model (45) leads to the appearance of a qualitatively new effect, i.e., the possible emergence of an $s+i s$ state that breaks time-reversal symmetry. Indeed, in the model (45), each band would like to have a gap of opposite sign with respect to the gap in the other bands: when three gaps compete, one then realizes a situation analogous to the antiferromagnet in the triangular lattice, where spins orient themselves at relative $2 \pi / 3$ angles. In the SC problem, the frustration occurs in the range of parameters (i.e., interactions and/or temperature) where two eigenvalues of the matrix $\hat{\Pi}-\hat{g}^{-1}$ vanish (see Sec. III B and Appendix C), allowing for an intrinsically complex SC order parameter. Since in this case $\Delta_{l}^{*} \neq \Delta_{l}$, time reversal (which corresponds to complex conjugation) is spontaneously broken, and the system is in an $s+i s$ TRSB state. To give a general idea of the range of parameters for the TRSB phase, we show in Fig. 3 the phase diagram of the specific model (45). Here, we assumed for simplicity that the DOS $N_{l} \equiv N$ in all bands are equal, so that we can introduce the dimensionless couplings

$$
\eta=V_{h h} N, \quad \lambda=V_{h e} N .
$$

As it has been discussed previously, ${ }^{17,24}$ the $T_{\text {TRSB }}$ separating the normal superconductor from the TRSB state ends at a finite value $\lambda_{\text {cr }}(\eta)>\eta$, while for $\lambda<\eta$, the TRSB phase is always present at $T=0$, but the $T_{\mathrm{TRSB}}$ is exponentially suppressed. By using $\eta=0.5$ and $\omega_{0}=15 \mathrm{meV}$ for the BCS bosonic 


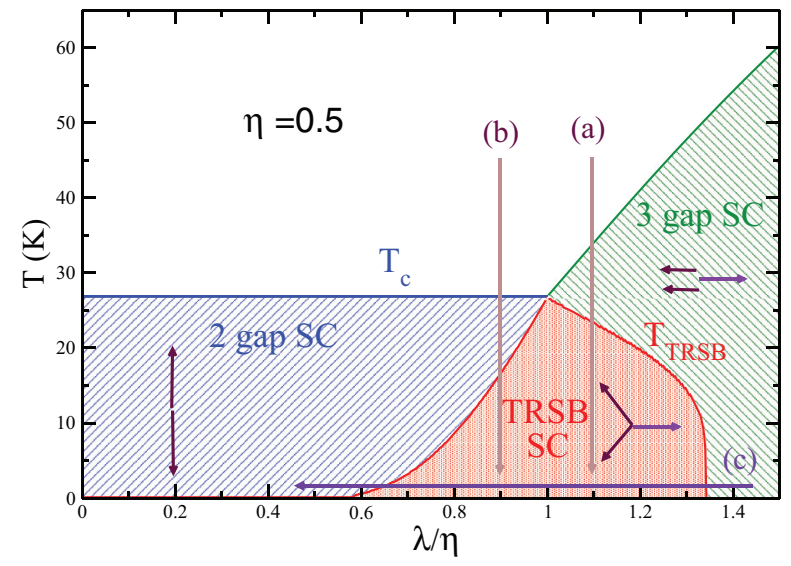

FIG. 3. (Color online) Phase diagram of the model (45) obtained by numerical solution of the mean-field equations (25). Here, we used as bosonic scale $\omega_{0}=15 \mathrm{meV}$. Notice that the $T_{\mathrm{TRSB}}$ line ends at a finite value of $\lambda / \eta$ for $\lambda>\eta$, while for $\lambda<\eta T_{\mathrm{TRSB}}$, it is, in principle, always finite but it is exponentially suppressed as one moves to small $\lambda$ values.

scale in the $\Pi_{l}$ bubbles, as roughly appropriate for pnictides, ${ }^{32}$ one obtains $\lambda_{\text {cr }} \simeq 1.3$, leading to a reasonable wide range of parameters where the TRSB transition can occur. Even though these numbers have to be considered only indicative for real materials, due to the simplifications of the model (45) and the overestimation of the critical temperatures in mean-field like calculations, for a specific sample, one could indeed observe one of the thermal transitions marked by vertical lines in Fig. 3.

As we discussed above, several other possibilities exist for an intermediate TRSB state in pnictides, depending on the nature of the competing SC channels, which reflects on the structure of the matrix (45) and on its eigenvalues. For example, to account for a possible $d$-wave symmetry in $\mathrm{KFe}_{2} \mathrm{As}_{2}$, one should include a third hole pocket with a large intraband-repulsion term. ${ }^{41}$ On the other hand, the same matrix structure (45) but with a different identification of the bands ${ }^{30}$ can be used to describe the $s+i d$ state that has been proposed for the electron-doped pnictides. ${ }^{30,49-51}$ In this case, by adding to the schematic structure of Fig. 2(a) an interband repulsion between the electron pockets, ${ }^{30,49}$ one could induce a SC state with a sign change of the gap between the $(0, \pi)$ and $(\pi, 0)$ pockets, which corresponds to $d$-wave symmetry, even if without nodes on the FSs. As we shall see below, our approach allows us to establish a general correspondence between the structure of mean-field equations (25) and the evolution of the collective modes, providing thus a general scheme to test experimentally whether or not a TRSB state is realized, regardless the specific symmetry of the two degenerate SC channels active in the TRSB phase. For this reason, while previous work has focused on the $T=0$ behavior of the collective modes as a function of the tuning parameter (i.e., the doping) for the quantum TRSB transition, here we focus on the possibility to identify the occurrence of a thermal TRSB transition. Indeed, while the quantum phase transition between the TRS and TRSB state has in general only two end points, the thermal transition occurs in a much wider range of parameters, making eventually its identification a more accessible experimental task.

\section{B. Collective mode across the TRSB transition}

To extend the collective-modes derivation of Sec. II, we will start by considering the most general three-band model which admits a TRSB state and has one antibonding SC channel. Thus $\hat{g}$ must have two positive and one negative eigenvalue, so that after the rotation $R$ the diagonal matrix $\hat{\Lambda}$ is

$$
\hat{g}=R^{-1}\left(\begin{array}{ccc}
\Lambda_{1} & 0 & 0 \\
0 & \Lambda_{2} & 0 \\
0 & 0 & -\Lambda_{3}
\end{array}\right) R
$$

where we also set by definition $\Lambda_{1} \geqslant \Lambda_{2}$. For example, in the simplified model (45), one has that for $\eta>\lambda$ is $\Lambda_{1}=\eta, \Lambda_{2}=$ $\left(\sqrt{\eta^{2}+8 \lambda^{2}}-\eta\right) / 2$, and $\Lambda_{3}=\left(\sqrt{\eta^{2}+8 \lambda^{2}}+\eta\right) / 2$, while for $\eta<\lambda$ the role of $\Lambda_{1}$ and $\Lambda_{2}$ is interchanged. The derivation of the effective action is then a straightforward generalization of the procedure used in Sec. II. In particular, also in this case, one has to introduce a HS field $h_{3}$ associated with the repulsive channel $\Lambda_{3}$, and one can take advantage of the generalized transformation $T$ (depending now on three parameters, see Appendix C) to impose $\bar{h}_{3}=0$ at the saddle point. Indeed, the equivalent of the saddle-point (20) can be made diagonal again by using the three conditions that generalize Eq. (22), i.e.,

$$
\sum_{l} T_{i l} \Pi_{l} T_{j \neq i, l}=0, \quad i, j=1,2,3,
$$

so that one is left with

$$
\left(\begin{array}{ccc}
\frac{1}{\Lambda_{1}}-\sum_{l} T_{1 l}^{2} \Pi_{l} & 0 & 0 \\
0 & \frac{1}{\Lambda_{2}}-\sum_{l} T_{2 l}^{2} \Pi_{l} & 0 \\
0 & 0 & -\frac{1}{\Lambda_{3}}-\sum_{l} T_{3 l}^{2} \Pi_{l}
\end{array}\right)\left(\begin{array}{c}
\bar{h}_{1} \\
\bar{h}_{2} \\
i \bar{h}_{3}
\end{array}\right)=Y_{1} \bar{h}_{1}+Y_{2} \bar{h}_{2}+i Y_{3} \bar{h}_{3}=0
$$

where the $Y_{i}$ are the eigenvalues of the matrix $\hat{g}^{-1}-\hat{\Pi}$, which enters the usual mean-field equations (25). As one can see, in full analogy with the two-band case (20) above, the coefficient $Y_{3}$ which multiplies the antibonding HS field $\bar{h}_{3}$ is always positive, so that one imposes $\bar{h}_{3}=0$ at the saddle point. The remaining two coefficients $Y_{1}$ and $Y_{2}$ can be both in principle vanishing, leading to finite saddle-point values of the corresponding HS fields.

Let us discuss the thermal evolution equivalent to one of the paths (a) and (b) in Fig. 3, starting from the non-SC state. As T decreases and the Cooper bubbles increase, the first coefficient that vanishes at $T_{c}$ in Eq. (49) is, for example, $Y_{1}$. Then $h_{1}$ is 
the first HS field that orders. Its phase can be chosen real, $\bar{h}_{1}=R_{1}$, so all the gaps are given by Eq. (24) and are real. As the temperature decreases further, according to the range of parameters of the matrix $\hat{g}$, it is possible that at $T=T_{\mathrm{TRSB}}$ also $Y_{2}\left(T_{\mathrm{TRSB}}\right)$ vanishes,

$$
Y_{2}\left(T_{\mathrm{TRSB}}\right)=\sum_{l} T_{2 l}^{2}\left(T_{\mathrm{TRSB}}\right) \Pi_{l}\left(T_{\mathrm{TRSB}}\right)-1 / \Lambda_{2}=0 .
$$

In this case, as we discuss in Appendix C, one can also show that at lower temperatures the imaginary part of $\bar{h}_{2}$ acquires a finite saddle-point value. More specifically, one can always choose a gauge where $\bar{h}_{2}$ is purely imaginary, i.e., $\bar{h}_{2}=i I_{2}$. As a consequence, the mean-field gaps at $T<T_{\mathrm{TRSB}}$ are given by

$$
\Delta_{l}=T_{1 l} \bar{h}_{1}+T_{2 l} \bar{h}_{2}=T_{1 l} R_{1}+i T_{2 l} I_{2}=\left|\Delta_{l}\right| e^{i \bar{\vartheta}_{l}},
$$

so that they are intrinsically complex and a TRSB state is reached. Moreover, the additional $Z_{2}$ symmetry between the two possible time-reversal-symmetry breaking ground states (51) is encoded in the complex conjugation for the $\bar{h}_{2}$ field, which leads to a change of sign of all the phases $\vartheta_{l}$ without changing the ground-state energy.

The emergence of a finite imaginary part of $\bar{h}_{2}$ below TRSB has a precursor effect on the behavior of the collective phase modes above $T_{\mathrm{TRSB}}$. Indeed, in the TRS phase where all the gaps have trivial phases, one can obtain a straightforward extension of Eqs. (35) and (36) for the amplitude and phase fluctuations of the HS fields. In particular, by using again the constraints (48) for the $T$ transformation, the equivalent of Eq. (39) for the phase sector $\eta_{q}^{T}=\left(\operatorname{Im} h_{1, q}, \operatorname{Im} h_{2, q}, i \operatorname{Im} h_{3, q}\right)$ in the long-wavelength $q \simeq 0$ limit can be written as

$$
\hat{S}_{\mathrm{FL}}^{P}(q=0)=\left(\begin{array}{ccc}
-\sum_{l} \Pi_{l} T_{1 l}^{2}+\frac{1}{\Lambda_{1}} & 0 & 0 \\
0 & -\sum_{l} \Pi_{l} T_{2 l}^{2}+\frac{1}{\Lambda_{2}} & 0 \\
0 & 0 & -\sum_{l} \Pi_{l} T_{3 l}^{2}-\frac{1}{\Lambda_{3}}
\end{array}\right) .
$$

Equation (52) is one of the central results of our paper. Indeed, it establishes a direct correspondence between the masses of the phase modes and the saddle-point equations (49), showing that as soon as one reaches the TRSB state, defined by Eq. (50), the fluctuations of the $\operatorname{Im} h_{2}$ HS field become massless. It must be emphasized that the this result holds regardless the structure of the coupling matrix. Indeed, one can prove (Appendix C) that necessary and sufficient condition to have gaps with nontrivial phases is that two eigenvalues of the matrix $\hat{g}^{-1}-\hat{\Pi}$, i.e., two $Y_{i}$ coefficients in the diagonal form (49), must vanish. Since the $T$ transformation decouples also the phase modes and connects their masses at $T \geqslant T_{\mathrm{TRSB}}$ to the $Y_{i}$ coefficients, it makes possible to show in full generality that at the boundary between a TRS and a TRSB phase one additional phase mode becomes massless. By considering then the phase diagram of Fig. 3, such a massless mode emerges along all the line $T_{\mathrm{TRSB}}$, as well as for isothermal transitions ${ }^{14,15,17}$ as a function of the coupling parameters for the matrix $\hat{g}$, like path (c). In this case, the TRSB state would be equally determined by the condition $Y_{2}=0$, considering $Y_{2}$ a function, e.g., of the SC coupling $\lambda$ :

$$
Y_{2}\left(\lambda_{\mathrm{TRSB}}\right)=\sum_{l} T_{2 l}^{2}\left(\lambda_{\mathrm{TRSB}}\right) \Pi_{l}\left(\lambda_{\mathrm{TRSB}}\right)-1 / \Lambda_{2}=0 .
$$

It is worth stressing that our derivation shows also that in the three-band case only one additional mode (other than the Bogoliubov-Anderson Goldstone mode) can be massless at the TRSB transition. Indeed, for interband-dominated coupling, the fluctuations of the antibonding $h_{3}$ field in Eq. (52) do not identify a mode, as explained in Sec. II. On the other hand, if also the third eigenvalue $\Lambda_{3}$ of the matrix (47) were positive, the associated $h_{3}$ fluctuations would describe a Leggett-like mode that cannot become massless, since at least one eigenvalue of the decomposition (49) must be finite (see Appendix C). The possibility to establish these results on general grounds is crucial to identify the total number of massless modes a priori. Indeed, an explicit numerical calculation of the collective modes, done, e.g., by using the low-frequency expansion of the bubbles, ${ }^{16,17}$ becomes very delicate when one of the gaps vanishes, as we shall discuss in more details in the next section.

Below $T_{\mathrm{TRSB}}$, the behavior of the collective modes is more complex, due to the mixing between amplitude and phase fluctuations. ${ }^{14,16,17}$ Indeed, when the SC gaps $\Delta_{l}$ in each band are complex numbers, the fermionic bubbles, which appear in Eqs. (35) and (36), acquire an explicit dependence on the saddle-point values $\bar{\vartheta}_{l}$ of the phases of the SC order parameters. More specifically, one has that

$$
\begin{gathered}
\Lambda_{l}^{11}(q)=\bar{\Lambda}_{l}^{22}(q)+2 \cos ^{2} \bar{\vartheta}_{l} F_{l}(q), \\
\Lambda_{l}^{22}(q)=\bar{\Lambda}_{l}^{22}(q)+2 \sin ^{2} \bar{\vartheta}_{l} F_{l}(q), \\
\Lambda_{l}^{12}(q)=2 \sin \bar{\vartheta}_{l} \cos \bar{\vartheta}_{l} F(q)+\mathcal{O}\left(q^{2}\right),
\end{gathered}
$$

where $\bar{\Lambda}_{l}^{22}$ is a function of $\left|\Delta_{l}\right|$, so it coincides with the expression (A7) of the $\Lambda^{22}$ bubble computed assuming a real gap, and

$$
F_{l}(q)=2\left|\Delta_{l}\right|^{2} \frac{T}{V} \sum_{\mathbf{k}, n} \frac{1}{\left(\Omega_{m}+\omega_{n}\right)^{2}+E_{\mathbf{k}+\mathbf{q}}^{2}} \frac{1}{\omega_{n}^{2}+E_{\mathbf{k}}^{2}}
$$

is also a function only of the gap amplitude $\left|\Delta_{l}\right|$. When the gaps have trivial phases $\bar{\vartheta}_{l}=0, \pi$, these definitions coincide with the ones given in Appendix $\mathrm{A}$ and one recovers the expansion (37) and (38) used above. Below $T_{\mathrm{TRSB}}$, the most important difference is that the bubbles $\Lambda_{l}^{12}$, which appear in the coupling between the amplitude and phase sectors [see Eq. (A5)] cannot be neglected, making the structure of the Gaussian fluctuations (27) considerably more complicated. 
In this situation, the structure of the collective modes is not simplified by the use of the transformation $T$. Thus, in order to simplify the numerical computation, we will take advantage of the fact that thanks to Eqs. (35) and (36) the overall action for mixed amplitude and phase fluctuations is a $6 \times 6$ matrix given by

$$
\begin{aligned}
\hat{S}_{\mathrm{FL}} & =\frac{1}{2}\left(\begin{array}{cc}
T \hat{\Lambda}^{11} T^{T}+2 \hat{\Lambda}^{-1} & T \hat{\Lambda}^{21} T^{T} \\
T \hat{\Lambda}^{12} T^{T} & T \hat{\Lambda}^{22} T^{T}+2 \hat{\Lambda}^{-1}
\end{array}\right) \\
& =\frac{1}{2} \hat{T}\left(\begin{array}{cc}
\Lambda^{11}+2 \hat{g}^{-1} & \hat{\Lambda}^{21} \\
\hat{\Lambda}^{12} & \hat{\Lambda}^{22}+2 \hat{g}^{-1}
\end{array}\right) \hat{T}^{T} \equiv \hat{T} \hat{M} \hat{T}^{T},
\end{aligned}
$$

where we used the property (9) that $\hat{\Lambda}^{-1}=T g^{-1} T^{T}$ and we defined $\hat{T}$ as a $6 \times 6$ diagonal matrix having the $3 \times 3$ matrix $T$ on the diagonal. Since $\operatorname{det} \hat{T}=1$, the collective modes will be given by the solutions of the equation $\operatorname{det} \hat{M}=0$. It is worth noting that the corresponding eigenvectors can be associated to amplitude and phase fluctuations in the various bands: indeed, the relations (33) and (34) between the fermionic operators and the HS fields will read in this case:

$$
\begin{aligned}
& \left\langle\operatorname{Re} \Delta_{l, q}\right\rangle=T_{l 1}\left\langle\operatorname{Re} h_{1, q}\right\rangle+T_{l 2}\left\langle\operatorname{Re} h_{2, q}\right\rangle+T_{l 3}\left\langle i \operatorname{Re} h_{3, q}\right\rangle, \\
& \left\langle\operatorname{Im} \Delta_{l, q}\right\rangle=T_{l 1}\left\langle\operatorname{Im} h_{1, q}\right\rangle+T_{l 2}\left\langle\operatorname{Im} h_{2, q}\right\rangle+T_{l 3}\left\langle i \operatorname{Im} h_{3, q}\right\rangle,
\end{aligned}
$$

which correspond in a short notation to, e.g., $\langle\operatorname{Re} \Delta\rangle=$ $T^{T}\langle\operatorname{Re} h\rangle$, with the usual inclusion of the imaginary unit in the fluctuations of the antibonding field $h_{3}$. Thus it is not surprising that the $\hat{M}$ matrix coincides with the derivation done in Refs. 16 and 17 by means of linear response theory in the band basis. In addition, in the case of dominant intraband pairing, where no imaginary unit is associated to the HS fields, the relations (59) and (60) can be used to define new bosonic variables. In this case, when all the gaps are opened so that $\operatorname{Im} \Delta_{l, q}=\Delta_{l} \vartheta_{l, q}$, by means of the identity (A29), one recovers for the phase sector the same structure reported in Ref. 15. Notice also that the coupling between fluctuations in different bands is provided by the inverse matrix $\hat{g}^{-1}$ of the SC couplings, while the coupling between the amplitude and phase sector is diagonal in the band index and it is given by the $\Lambda_{l}^{12}$ bubbles of Eq. (56), which are proportional to the $\sin \bar{\vartheta}_{l}$, so that they differ from zero only in the TRSB state. This result is very general, and indeed it can be found also within the phenomenological multiband Ginzburg-Landau approach of Ref. 14, where the interband couplings are provided by Josephson-like terms.

\section{Temperature and coupling dependence of the Leggett mode}

To show explicitly the temperature evolution of the Leggett modes, we will refer for simplicity to the set of coupling constants defined by Eq. (45), which gives rise to the phase diagram shown in Fig. 3. As one can see, while Eq. (58) does not allow for a simple identification of the number and nature of the collective modes, it simplifies the numerical evaluation of the modes since one does not need to determine also the $T$ matrix. We then solved self-consistently the gap equations and computed the matrix $\hat{M}$ in Eq. (58), looking for well-defined modes below the threshold $2 \Delta_{\text {min }}$ provided by the smallest gap in the problem. We assume conventionally that the gap in the
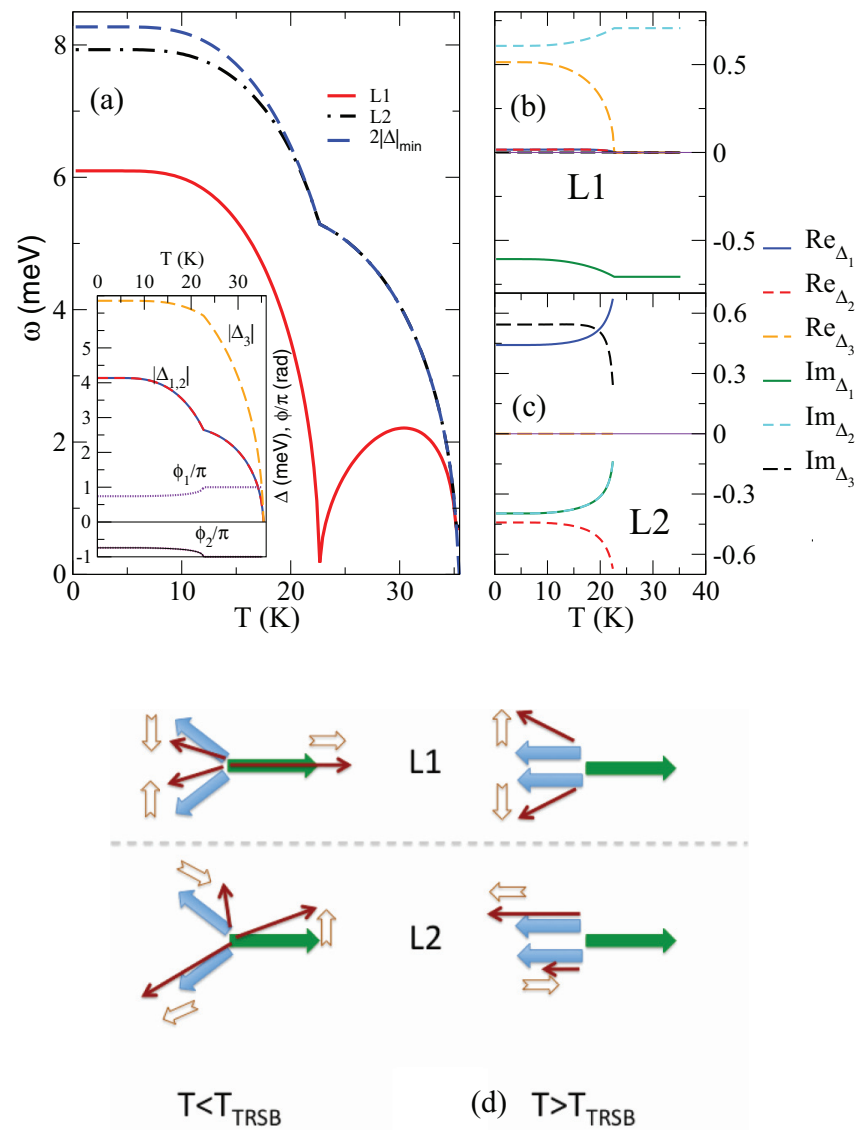

FIG. 4. (Color online) Temperature evolution of the low-energy modes along the path (a) of Fig. 3 , corresponding to $\lambda=1.12 \eta$. Here, $T_{c}=35.5 \mathrm{~K}$ and $T_{\mathrm{TRSB}}=23 \mathrm{~K}$. (a) Temperature dependence of the low-energy mode along with the minimum gap threshold, obtained by the temperature dependence of the gaps reported in the inset along with the phases of the hole gaps. (b) and (c) components of the eigenvectors corresponding to the modes labeled as L1 and L2 in (a). (d) Schematic structure of the modes below and above $T_{\mathrm{TRSB}}$. Here, big full arrows denote the equilibrium gaps, while the thin arrows denote the gaps including the fluctuations, identified by the big empty arrows. As one can see, L1 evolves in the ordinary Leggett mode for the order parameters in the two hole bands, while L1 evolves towards an amplitude mode.

electron band $\Delta_{3}$ is real and positive, while the gaps in the hole bands are $\Delta_{1}=\Delta e^{i \phi_{1}}, \Delta_{2}=\Delta e^{i \phi_{2}}$. According to the phase diagram of Fig. 3 , the three phases correspond respectively to

$$
\begin{gathered}
3 \text { gaps }-\mathrm{SC}: \Delta_{3} \neq 0, \quad \phi_{1}=\phi_{2}=\pi, \\
\text { TRSB }-\mathrm{SC}: \Delta_{3} \neq 0, \quad \phi_{1}=\phi, \quad \phi_{2}=-\phi, \\
2 \text { gaps }-\mathrm{SC}: \Delta_{3}=0, \quad \phi_{1}=\pi / 2, \quad \phi_{2}=-\pi / 2 .
\end{gathered}
$$

Let us start from the path labeled by (a) in Fig. 3, see Fig. 4. Here, we identify a mode L1 that softens at the $T_{\mathrm{TRSB}}$ and remains always below the gap threshold. Above $T_{\mathrm{TRSB}}, \mathrm{L} 1$ is an ordinary Leggett mode associated to the phase fluctuations in the two hole bands. Indeed, in this state, the pairing in each hole band is provided by the interband coupling to the third electron band. Thus, within the hole-bands sector, the problem is formally equivalent to a two-gaps superconductor 
with dominant intraband pairing, and the Leggett mode is well defined. Below the $T_{\mathrm{TRSB}}$, the SC order parameter in the hole bands becomes complex, so that the Leggett-like oscillation drives also amplitude fluctuations both in the hole and electron bands. Observe that below $T_{\mathrm{TRSB}}$ a second low-energy mode appears, labeled L2 in Fig. 4, which is only slightly below the gap threshold. Indeed, at $T>T_{\mathrm{TRSB}}$, this mode coincides with pure amplitude fluctuations in the two hole bands, and thus it appears right at the gap edge $2 \Delta_{1,2} \equiv 2 \Delta_{\min }$. However, as one moves at higher $\lambda / \eta$ values or one makes the two hole pockets inequivalent, this mode approaches rapidly the gap edge, becoming then overdamped. On the other hand, at the full symmetric point $\lambda=\eta, \mathrm{L} 1$ is exactly degenerate with the $\mathrm{L} 2$ mode. Indeed, at the $\lambda=\eta$ point, the three bands are completely equivalent, and the L1 and L2 describe the same oscillation: the gaps in two bands approach each other, inducing a change of modulus of the third gap. ${ }^{16}$

When one moves in the regime $\lambda<\eta$ [path (b) in Fig. 3], the role of the two modes in the TRSB state changes and L2 becomes softer. More interestingly, at $T>T_{\mathrm{TRSB}}$, the situation is completely different in this case, since no soft mode can be found. This result can be easily understood: at $T>T_{\mathrm{TRSB}}$, the gap in the electron band closes and the system is formally equivalent to a two-band superconductor with dominant interband coupling. This is the situation discussed in Sec. II, where no Leggett-like mode is present since only one bonding SC channel exists. By close inspection of the eigenvector components in Figs. 5(b)-5(d), one sees that as $T \rightarrow T_{\mathrm{TRSB}}^{-}$, the $\mathrm{L} 2$ mode tends to the Goldstone mode, while the L1 mode would coincide to the ordinary Leggett oscillation, which does not identify a mode above $T_{\mathrm{TRSB}}$ for the reason explained above. We then recover the same result discussed below Eq. (52) in the language of the $h_{1,2}$ fields, for example, exactly at $T=T_{\mathrm{TRSB}}$, there are two solutions at $\omega=0$. However, while the Goldstone mode is always well defined and it remains massless, the other solution can be connected to a well-defined mode only below $T_{\mathrm{TRSB}}$, where all the three gaps are opened. In this respect, as soon as one modifies slightly the coupling matrix (45) in order to make the two hole pockets inequivalent, the gap in the electronic band in general survive up to $T_{c}$. In this case, a soft mode can be found also in the whole temperature interval $T_{\mathrm{TRSB}}<T<T_{c}$, with a similar temperature dependence as the one shown in Fig. 4.

It is worth noting that in evaluating numerically the collective modes, we retained the full frequency dependence of the electronic bubbles in Eq. (58). Indeed, the close proximity of one of the soft modes in the TRSB phase to the gap edge makes the low-frequency expansion ${ }^{16,17}$ dangerous, as observed also in Ref. 17. This is shown explicitly in Fig. 6, where we report the temperature dependence of the collective modes obtained by using the low-frequency approximation (37) and (38) of the fermionic bubbles that appear in Eq. (58). As one can see, while the massless character of the $h_{2}$ fluctuations is correctly recovered at $T=T_{\mathrm{TRSB}}$, the absolute value of the low-energy modes in the TRSB state is completely wrong in this approximation. In particular, in the case $\eta<\lambda$ [see Fig. 6(b)], no mode is found below the threshold for the quasiparticle excitations. Even adding the next-order term in the low-frequency expansion (37), as suggested in Ref. 17,
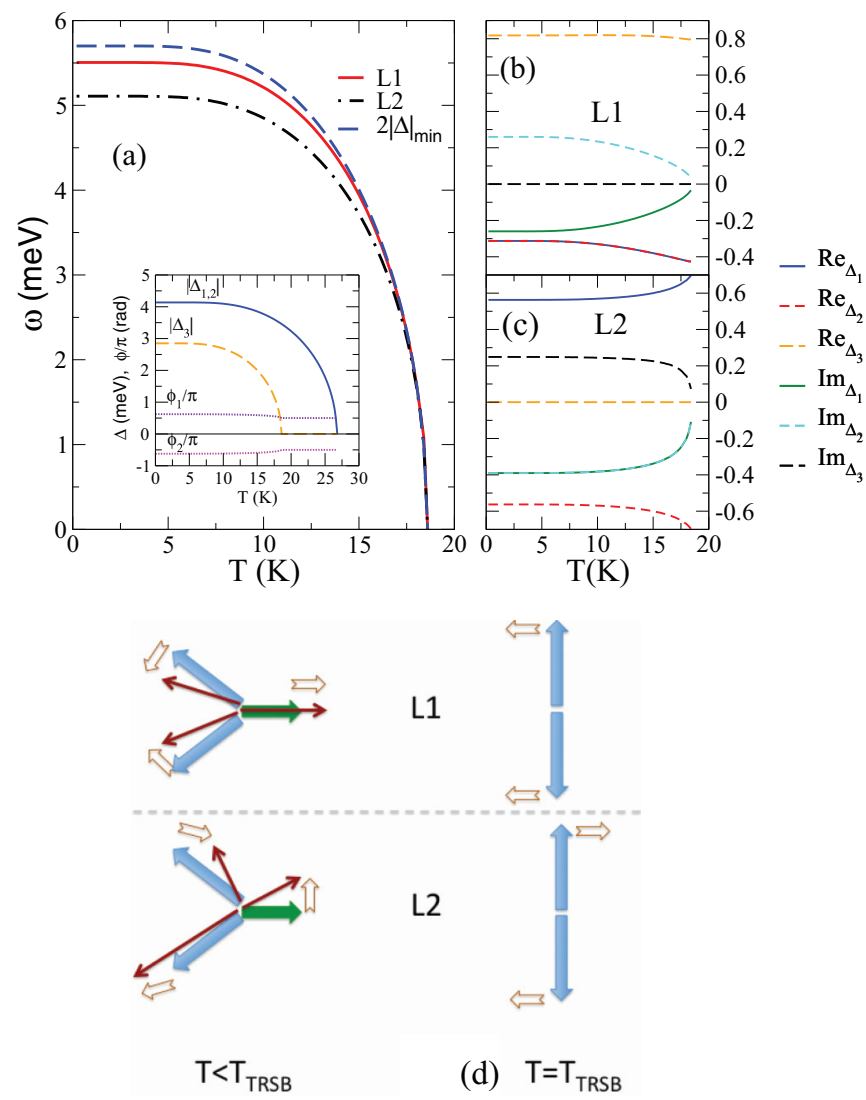

FIG. 5. (Color online) Temperature evolution of the low-energy modes along the path (b) of Fig. 3, corresponding to $\lambda=0.92 \eta$. Here, $T_{c}=23 \mathrm{~K}$ and $T_{\mathrm{TRSB}}=18.6 \mathrm{~K}$. (a) Temperature dependence of the low-energy mode along with the minimum gap threshold, obtained by the temperature dependence of the gaps reported in the inset along with the phases of the hole gaps. (b) and (c) Component of the eigenvectors corresponding to the modes labeled as L1 and L2 in (a). (d) Schematic structure of the modes below and above $T_{\text {TRSB }}$, with the notation of Fig. 4. In contrast to the case $\lambda>\eta$ shown in Fig. 4, here the fluctuations above $T_{\mathrm{TRSB}}$ do not identify a mode. Nonetheless, exactly at $T=T_{\mathrm{TRSB}}$, L2 coincides with the Goldstone mode, while L1 appears as an ordinary Leggett-like oscillation of the gaps in the two hole bands.

only one mode moves below the gap edge, in contrast to the correct result [see Fig. 5(a)].

To make also a closer connection to previous work, ${ }^{15-17}$ we show in Fig. 7 the evolution of the low-energy mode across the quantum TRSB transition, i.e., the path labeled with (c) in Fig. 3. Here [see Fig. 7(a)], the crossing between L1 and L2 at $\lambda=\eta$ is evident, and it is also clear that in the regime $\lambda<\eta$, the vanishing of the electronic gap will make it more difficult to resolve experimentally the soft mode. Indeed, even if $\omega_{L 1}$ remains well below the gap in the hole bands, it rapidly approaches $2 \Delta_{\min } \equiv 2 \Delta_{3}$. As we discussed above, this mode is missing in Ref. 17 since the authors used a low-energy expansion of the fermionic bubbles in the numerical evaluation of the collective modes. On the other hand, as soon as one makes the hole pockets inequivalent, ${ }^{17}$ the TRSB state admits an end point at a finite critical value $\lambda_{c}^{\min }$ also for $\eta<\lambda$, so that all the gaps are finite at $T_{\mathrm{TRSB}}$ and only one mode becomes massless at $\lambda_{c}^{\min }$. 

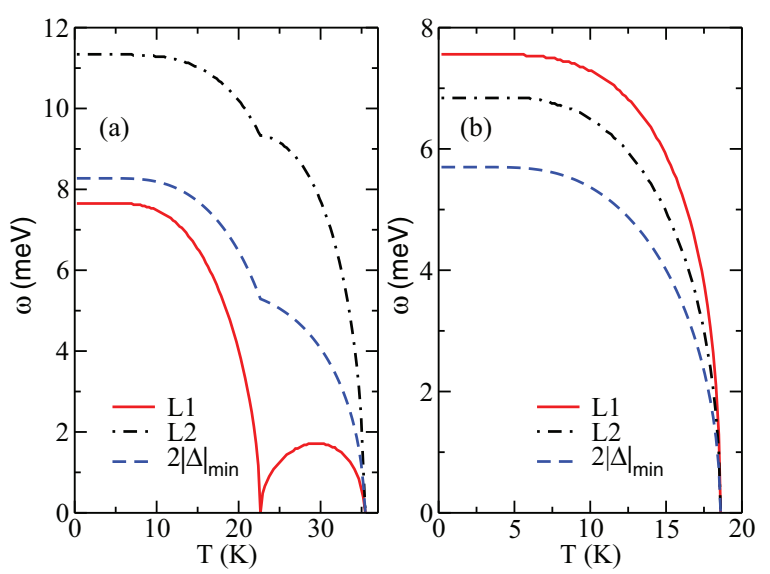

FIG. 6. (Color online) Evaluation of the collective modes by means of a low-frequency expansion of the fermionic bubbles for the case (a) $\lambda=1.12 \eta$ and (b) $\lambda=0.92 \eta$, whose exact solutions are reported in Figs. 4 and 5, respectively. Notice that in both cases, the frequencies of the low-energy modes are largely overestimated, and no soft mode is found for $\lambda=0.92$, in contrast to the correct result.

\section{Experimental signatures of the Leggett mode}

Let us discuss now the relevance of the present results to the experimental investigation of a TRSB state in pnictides. A natural probe for the identification of low-energy phase mode is Raman spectroscopy, in full analogy with the case of the intraband dominated superconductor $\mathrm{MgB}_{2} \cdot{ }^{22,23}$ Even though a full calculation of the Raman response is beyond the scope of the present manuscript, by following the results of several previous works, ${ }^{15,19,23,51}$ we outline the basic mechanism which can make phase modes visible in Raman. Raman scattering allows one to measure the response function for a charge density $\tilde{\rho}(\mathbf{q})=\sum_{\mathbf{k}} \gamma_{\mathbf{k}} \rho(\mathbf{q})$ weighted with a structure
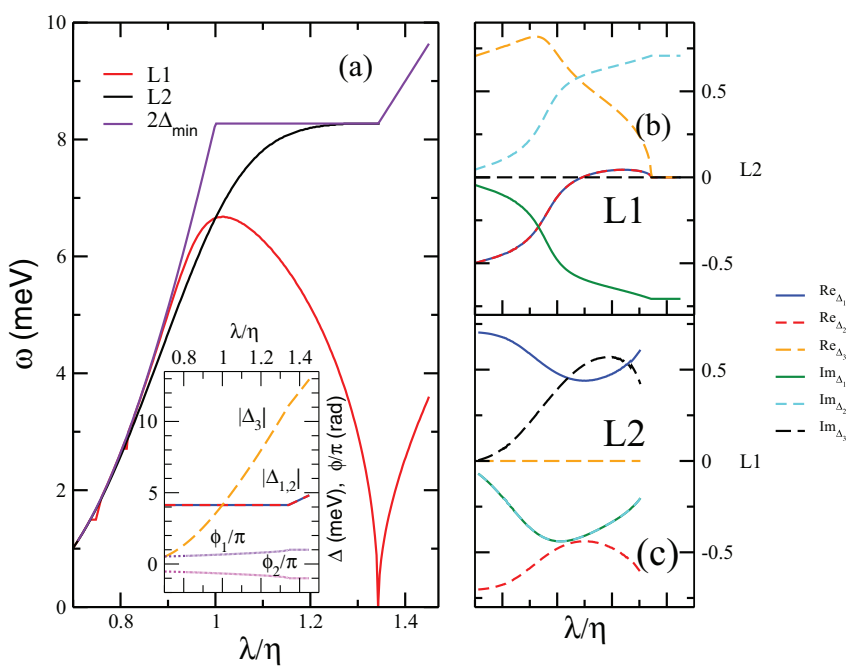

FIG. 7. (Color online) (a) Evolution of the low-energy modes along the path (c) of Fig. 3, i.e., as a function of the ratio $\lambda / \eta$ at $T=0$. The corresponding gap and phase values are shown in the inset. (b) and (c) Eigenvectors components of the two modes. Notice that at $\lambda=\eta$, the L1 and L2 mode are degenerate, as already observed before. ${ }^{16,17}$ On the other hand, as soon as one moves away from the symmetric point, one mode moves rapidly towards the gap edge. factor $\gamma_{\mathbf{k}}$ that accounts for the specific geometry of the incoming/outgoing light polarization. Since density and phase fluctuations are conjugate variables, $, 6,9,11,15$ phase fluctuations couple to the Raman response as well, with some caveats on the allowed symmetry for multiband superconductors. ${ }^{23}$ In practice, this means that on top of the bare Raman response due to quasiparticle excitations, that vanishes below $2\left|\Delta_{\min }\right|$ at $T \ll T_{c}$, collective phase excitations manifest themselves in the Raman response as a peak at the typical frequency $\omega_{L}$ of the corresponding modes. ${ }^{15,23,51}$ More specifically, when $\omega_{L}$ lies below the treshold $2\left|\Delta_{\min }\right|$ for quasiparticle excitations the mode at low $T$ is weakly damped by residual impurity-induced scattering processes, so the peak is sharp. In the specific case of pnictides, we have shown that Fig. 4 represents the typical thermal evolution of the phase modes across the transition from the TRS to the TRSB state, see path (a) of Fig. 3. Since the Leggett-like mode that becomes massless at the $T_{\mathrm{TRSB}}$ lies always below $2\left|\Delta_{\min }\right|$, it should be visible already when entering the normal SC state, with a nonmonotonic temperature dependence: it first softens until $T_{\mathrm{TRSB}}$ is reached and then hardens again, saturating at low $T$, see $\omega_{L 1}(T)$ in Fig. 4. Observe that in Fig. 4, the $\mathrm{L} 1$ mode involves above $T_{c}$ fluctuations in the two hole bands, which are assumed here for simplicity to have the same DOS, so that the interaction anisotropy can be tuned by a simple parameter $\lambda / \eta$. However, in real materials, the two hole bands have different DOS, and then different weighting factors $\gamma_{\mathbf{k}}^{l}$, which are simply proportional to the band masses. According to the discussion of Ref. 23, this guarantees that the L1 mode will be visible in Raman, despite that it involves phase fluctuations between bands having the same character.

We also verified that even in the region at $\lambda>\lambda_{\text {cr }} \simeq 1.3$ of the phase diagram of Fig. 3, where the TRSB transition does not explicitly occur, the Leggett mode lies well below the gap treshold $2 \Delta_{\min }$ in a wide range of temperatures and couplings, as shown also by the $T=0$ results reported in Fig. 7 . Thus, if in realistic materials with more anisotropic interactions, the phase space where a TRSB state is realized will shrink, ${ }^{17}$ making more difficult to realize a sample that displays the $s+i s$ state, its proximity can be still evidenced by the emergence of a phase mode at low (but finite) energy. In this situation, one can also investigate the effects of the Leggett mode on other quantities, as, for example, the superfluid density. Indeed, by expanding the phase-only action (52) at low frequency, one finds that the Goldstone mode and the Leggett one are coupled at finite frequency [see, e.g., Eq. (41)]. Thus, whenever the Leggett mode is massive it couples to fluctuations of the overall SC phase, which in turn, can affect the temperature dependence of the superfluid density, ${ }^{7,11}$ which has been recently shown to be highly nonmonotonic when 122 systems are strongly hole doped. ${ }^{44}$ This issue, which requires to account properly also for density fluctuations and long-range Coulomb interactions, ${ }^{11}$ not included so far, will be the subject of a future investigation.

\section{DISCUSSION AND CONCLUSIONS}

In the present manuscript, we analyzed the behavior of collective phase and amplitude modes in a multiband superconductor with predominant interband pairing, which is the case physically relevant for pnictide superconductors. The 
interband nature of the pairing mixes bonding (attractive) and antibonding (repulsive) SC channels, which must be treated with care while deriving the effective action for the SC fluctuations by means of the standard Hubbard-Stratonovich decoupling. Here, we implement a generalized transformation $T$ of the multiband pairing operators, which has two crucial consequences. (1) It allows to put the mean-field equations in a diagonal form:

$$
\left(\hat{g}^{-1}-\hat{\Pi}\right) \vec{\Delta}=0 \Rightarrow\left(\hat{\Lambda}^{-1}-T \hat{\Pi} T^{T}\right) \overrightarrow{\vec{h}} \equiv \sum_{l} Y_{l} \bar{h}_{l}=0 .
$$

The saddle-point values of the HS fields $\overrightarrow{\vec{h}}$ characterize the SC state. In the ordinary SC phase, the eigenvalue $Y_{1}=0$ connected to the largest SC bonding channel vanishes, and the saddle-point value $\bar{h}_{1}$ of the corresponding HS field is finite and can be taken real. The eigenvalue connected to the antibonding SC channel is always positive, so that the corresponding HS field is always zero. In this way, one sees that antibonding channels do not contribute to the SC state. A TRSB phase in the three-band model occurs when a second bonding SC channel becomes active, so that the corresponding eigenvalue, say $Y_{2}$, vanishes. In this situation, the corresponding imaginary part of the HS fields, $\bar{h}_{2}$ acquires a finite saddle-point value, and the gaps are intrinsically complex:

$$
\begin{gathered}
Y_{1}=0 \Rightarrow \mathrm{TRS}-\mathrm{SC}, \quad \bar{h}_{1}=R_{1}, \\
Y_{1}=Y_{2}=0 \Rightarrow \mathrm{TRSB}-\mathrm{SC}, \quad \bar{h}_{1}=R_{1}, \quad \bar{h}_{2}=i I_{2} .
\end{gathered}
$$

(2) The low-energy behavior of the collective phase fluctuations is uniquely determined by the mean-field equations. More specifically, one sees that at $q=0$ the fluctuations in the TRS phase sectors are described as

$$
S_{\mathrm{FL}}^{P}(q=0) \sim \sum_{l} Y_{l}\left(\operatorname{Im} h_{l}\right)^{2} .
$$

This has several implications: (i) the fluctuations of the ordering field $\operatorname{Im} h_{1}$ are trivially massless $\left(Y_{1}=0\right)$ since this is the Goldstone mode of the SC transition; (ii) for the two-band case, the fluctuations on the antibonding HS fields do not identify any collective mode, so that for interband-dominate pairing the Leggett mode is absent, in contrast to ordinary intraband-dominated superconductors as $\mathrm{MgB}_{2}$; (iii) in the three-band case, the transition to a TRSB phase $\left(Y_{2}=0\right)$ is uniquely associated to massless phase fluctuations described by the imaginary part of the ordering field $h_{2}$.

The absence of the Leggett mode in a two-band model for pnictides can be understood on physical grounds by having in mind the marked difference between a two-band superconductor with dominant intra- or interband pairing. In the former case, one has two bonding SC channels: the SC transition is controlled as usual by the one giving rise to a larger condensation energy, i.e., larger gaps. On the other hand, there exists a second possible solution with smaller gap values: the Leggett mode describes indeed deviations from the ground state in the direction of this second possible solution. For example, when the interband pairing is positive, the two gaps $\Delta_{1}, \Delta_{2}$ have the same sign, i.e., the same saddle-point phases $\bar{\vartheta}_{1}=\bar{\vartheta}_{2}$. In this situation, the Leggett mode identify phase fluctuations of opposite sign in the two bands, which would lead the system towards the solution $\bar{\vartheta}_{1}=-\bar{\vartheta}_{2}$ with higher energy, as reflected in the massive character of the Leggett mode. In this respect, antiphase oscillations can be put in resonance with the additional SC channel, so that they identify a collective mode. On the other hand, when the coupling is predominantly interband, there is no additional SC channel in the problem, so that the same kind of oscillations do not identify any proper mode of the system.

For the three-band case, it must be emphasized that the massless character of the Leggett phase mode found in item (2) above holds regardless the specific nature of the two SC bonding channels, which become degenerate at the TRSB transition. Thus, it can be applied as well also to other (multiband or multichannel) systems where a TRSB phase as been predicted, such as, e.g., highly doped graphene, ${ }^{52-54}$ water-intercaled sodium cobaltates, ${ }^{55}$ and locally noncentrosymmetric SrPtAs. ${ }^{56}$ The last system is particularly promising in this respect, since recent muon spin rotation $(\mu \mathrm{SR})$ measurements could be actually interpreted as evidence of a TRSB phase. ${ }^{57}$ Indeed, the massless character of the phase mode at the transition is a unique signature of the TRSB transition that can be used to rule out other possible interpretation of the $\mu \mathrm{SR}$ data.

In the specific case of pnictides, by performing an explicit numerical calculation of the collective modes, we also established some additional results. In particular, we found that below $T_{\mathrm{TRSB}}$, the massless mode becomes massive again, while a second low-energy mode can appear in some range of parameters. For the case of pnictides, by scanning in temperature a given sample that is either in case (a) or (b) of Fig. 3, the appearance of a mode below the gap threshold will signal the emergence of the TRSB state. Since phase fluctuations couple to density fluctuations, ${ }^{11,15,18,19,23}$ the most direct probe of this mode is via Raman scattering, in analogy with the result found for $\mathrm{MgB}_{2} .{ }^{22}$ Even though a full calculation of the Raman response is beyond the scope of the present manuscript, we expect that in the realistic case of pnictides, the temperature evolution of the Raman spectra can offer a powerful mean to the identification of a TRSB state, shedding then new light on the theoretical understanding of the pairing mechanism itself in these unconventional superconductors.

\section{ACKNOWLEDGMENTS}

We thank S. Caprara for useful discussions and suggestions. The authors acknowledge financial support by the Italian MIUR under the project FIRB-HybridNanoDevRBFR1236VV and by the Spanish Ministerio de Economía y Competitividad (MINECO) under the project FIS2011-29680.

\section{APPENDIX A: EQUIVALENCE BETWEEN THE GAUSSIAN ACTION DERIVED IN CARTESIAN OR POLAR COORDINATES}

In this Appendix, we show that in the single-band case the Gaussian action for amplitude and phase fluctuations can be equally derived by using a Cartesian (real and imaginary parts) or polar (amplitude and phase) description of the SC fluctuations. In the multiband case, as we discussed in the 
present manuscript, one chooses as convenient variables (i.e., as HS fields) proper combinations of the gaps in the various bands. Nonetheless, when all the SC channels are bonding, one can still introduce a set of HS fields associated to the fluctuations in each band. Indeed, in this case, the equivalent of the relations (33) and (34) between the physical fields and the HS fields do not contain imaginary units. Thus they are not only valid for the average values, but they represent a change of variables for bosonic fields in the functional integral defining the partition function. This case will be discussed indeed in Appendix B, where we explicitly apply our formalism to the two-band case with predominant intraband pairing. However, as soon as at least one channel is antibonding, the only possible route is to use collective HS fields properly defined in each channel, as we have done in the present manuscript. Still, the general relations between the fermionic bubbles that we will establish below can be used to interpret the low-energy behavior of the collective modes. In particular, it will be useful to compare the derivation of collective modes in the TRSB phase given in Refs. 13 and 15, based on a polar description, and the one presented here, based on the use of Cartesian coordinates, equivalent also to the approach of Refs. 16 and 17.

Let us start again from Eq. (1) written for a single-band superconductor, and let us decouple the interaction term by means of the HS decoupling (5), by introducing an HS field $\Delta(x)$. The equivalent of Eq. (12) will then read

$$
S=S_{0}+\int d \tau d \mathbf{x} \frac{|\Delta(x)|^{2}}{g}-\left(\Delta^{*} c_{\downarrow} c_{\uparrow}+\text { H.c. }\right) .
$$

After integration of the fermions the saddle-point value $\Delta$ (chosen to be real) of the HS field will appear in the mean-field Green's function $\bar{G}$, while its fluctuations will be decomposed as $\Delta_{\mathbf{q}}=\operatorname{Re} \Delta_{\mathbf{q}}+i \operatorname{Im} \Delta_{\mathbf{q}}$, so that Eqs. (14) and (15) will be explicitly given by

$$
\begin{aligned}
\bar{G}_{k}^{-1} & =\left(\begin{array}{cc}
i \omega_{n}-\xi_{k} & \Delta \\
\Delta & i \omega_{n}+\xi_{k}
\end{array}\right), \\
\Sigma_{q=k-k^{\prime}} & =\sqrt{\frac{T}{V}}\left(\operatorname{Re} \Delta_{q} \sigma_{1}+\operatorname{Im} \Delta_{q} \sigma_{2}\right),
\end{aligned}
$$

where $\Delta$ is the solution of the self-consistency equation:

$$
\Pi=\frac{1}{g} \Rightarrow \frac{1}{V} \sum_{\mathbf{k}} \tanh \frac{\beta E_{\mathbf{k}}}{2} \frac{1}{2 E_{\mathbf{k}}}=\frac{1}{g},
$$

where $\Pi$ is the Cooper bubble (21) and $E_{\mathbf{k}}=\sqrt{\xi_{\mathbf{k}}^{2}+\Delta^{2}}$. The explicit connection established by Eq. (A3) above between the real and imaginary part of the fluctuating field and the $\sigma_{1}, \sigma_{2}$ Pauli matrices, respectively, allows one to derive immediately the coefficients of the action $S_{\mathrm{FL}}(18)$ at a Gaussian order:

$$
S_{\mathrm{FL}}^{\mathrm{Car}}=\frac{1}{2} \sum_{q} \eta_{-q}^{T}\left(\begin{array}{cc}
\Lambda^{11}(q)+\frac{2}{g} & \Lambda^{21}(q) \\
\Lambda^{12}(q) & \Lambda^{22}(q)+\frac{2}{g}
\end{array}\right) \eta_{q},
$$

where in analogy with Eq. (27), we defined $\eta_{q}^{T}=$ $\left(\operatorname{Re} \Delta_{q}, \operatorname{Im} \Delta_{q}\right)$. The fermionic bubbles are defined as

$$
\Lambda^{i j}(q)=\frac{T}{V} \sum_{k} \operatorname{Tr}\left(\bar{G}_{k+q} \sigma^{i} \bar{G}_{k} \sigma^{j}\right) .
$$

More specifically, we have

$$
\begin{aligned}
\Lambda^{i j}(q)= & \frac{1}{V} \sum_{\mathbf{k}}\left[\frac{(u u)_{i j}}{E_{\mathbf{k}}^{\prime}-E_{\mathbf{k}}-i \Omega_{m}}+\frac{(v v)_{i j}}{E_{\mathbf{k}}^{\prime}-E_{\mathbf{k}}+i \Omega_{m}}\right] \\
& \times\left[f\left(E_{\mathbf{k}}^{\prime}\right)-f\left(E_{\mathbf{k}}\right)\right] \\
& +\left[\frac{(u v)_{i j}}{E_{\mathbf{k}}^{\prime}+E_{\mathbf{k}}-i \Omega_{m}}+\frac{(v u)_{i j}}{E_{\mathbf{k}}^{\prime}+E_{\mathbf{k}}+i \Omega_{m}}\right] \\
& \times\left[f\left(E_{\mathbf{k}}^{\prime}\right)-f\left(-E_{\mathbf{k}}\right)\right],
\end{aligned}
$$

where $E_{\mathbf{k}}^{\prime}=E_{\mathbf{k}+\mathbf{q}}$ and the coherence factors are given by ${ }^{39}$

$$
\begin{gathered}
(u u)_{11}=(v v)_{11}=\frac{1}{2}\left(1-\frac{\xi_{\mathbf{k}}^{\prime} \xi_{\mathbf{k}}-\Delta^{2}}{E_{\mathbf{k}}^{\prime} E_{\mathbf{k}}}\right), \\
(u v)_{11}=(v u)_{11}=\frac{1}{2}\left(1+\frac{\xi_{\mathbf{k}}^{\prime} \xi_{\mathbf{k}}-\Delta^{2}}{E_{\mathbf{k}}^{\prime} E_{\mathbf{k}}}\right), \\
(u u)_{22}=(v v)_{22}=\frac{1}{2}\left(1-\frac{\xi_{\mathbf{k}}^{\prime} \xi_{\mathbf{k}}+\Delta^{2}}{E_{\mathbf{k}}^{\prime} E_{\mathbf{k}}}\right), \\
(u v)_{22}=(v u)_{22}=\frac{1}{2}\left(1+\frac{\xi_{\mathbf{k}}^{\prime} \xi_{\mathbf{k}}+\Delta^{2}}{E_{\mathbf{k}}^{\prime} E_{\mathbf{k}}}\right), \\
(u u)_{12}=-(v v)_{12}=\frac{1}{2}\left(\frac{\xi_{\mathbf{k}}}{E_{\mathbf{k}}}-\frac{\xi_{\mathbf{k}}^{\prime}}{E_{\mathbf{k}}^{\prime}}\right), \\
(u v)_{12}=-(v u)_{12}=-\frac{1}{2}\left(\frac{\xi_{\mathbf{k}}^{\prime}}{E_{\mathbf{k}}^{\prime}}+\frac{\xi_{\mathbf{k}}^{\prime}}{E_{\mathbf{k}}^{\prime}}\right) .
\end{gathered}
$$

As one can easily check, $\Lambda^{12}(q) \sim \Omega_{m} \mathcal{O}\left(q^{2}\right)$, so that in the static limit it can be neglected, leading to the effective decoupling between the amplitude and phase fluctuations that has been used in Sec. II B. Moreover, from Eqs. (A7), (A10), and (A11) above, it follows that

$$
\Lambda^{22}(0)=-\frac{1}{V} \sum_{\mathbf{k}} \tanh \frac{\beta E_{\mathbf{k}}}{2} \frac{1}{E_{\mathbf{k}}}=-2 \Pi=-\frac{2}{g},
$$

where we used the self-consistency equation (A4) above. Thus one immediately recovers the massless character of the $\operatorname{Im} \Delta_{q}$ fluctuations from Eq. (A5). Notice that since we did not introduce explicitly the density fluctuations, other phase modes like the Carlson-Goldman one ${ }^{10,21,58}$ cannot be explicitly obtained. On the other hand, these sound-like modes are usually relevant only at high temperature or strong disorder, ${ }^{21}$ unless the gap has nodes, ${ }^{10}$ that are not the cases relevant for the present discussion.

Let us now discuss the derivation of the Gaussian fluctuations within the polar-coordinate scheme. ${ }^{11}$ In this case, before integrating our the fermions in Eq. (A1), one can make explicit the dependence on the phase $\theta$ of the HS field by means of a Gauge transformation on the fermionic fields, $c_{\sigma}(x) \rightarrow c_{\sigma}(x) e^{i \theta / 2}$. As a consequence, while $\bar{G}_{k}$ is unchanged, the self-energy $\Sigma_{k k^{\prime}}$ describing the SC fluctuations will be expressed in terms of the variables $|\Delta|_{q}, \theta_{q}:{ }^{11}$

$$
\begin{aligned}
\Sigma_{k k^{\prime}}= & \frac{T}{V}|\Delta|_{k-k^{\prime}} \sigma_{1}+\sqrt{\frac{T}{V}} \frac{i}{4 m}\left(\mathbf{k}-\mathbf{k}^{\prime}\right) \cdot\left(\mathbf{k}+\mathbf{k}^{\prime}\right) \theta_{k-k^{\prime}} \sigma_{0} \\
& +\sqrt{\frac{T}{V}} \frac{\omega_{k-k^{\prime}}}{2} \theta_{k-k^{\prime}} \sigma_{3} \\
& +\frac{T}{V} \sum_{\mathbf{s}} \frac{(\mathbf{k}-\mathbf{s}) \cdot\left(\mathbf{s}-\mathbf{k}^{\prime}\right)}{8 m} \theta_{k-s} \theta_{s-k^{\prime}} \sigma_{3}
\end{aligned}
$$


As one can see, in this case, the self-energy contains only spatial and time derivatives of the phase, associated, respectively, to the Pauli matrices $\sim \mathbf{k} \sigma_{0}$ and $\sigma_{3}$, which describe in Nambu formalism the fermionic current and density. Thus, using Eq. (A15) in the expansion (18), one can write the effective Gaussian action as

$$
S_{\mathrm{FL}}^{\mathrm{Pol}}=\frac{1}{2} \sum_{q} \zeta_{-q}^{T}\left(\begin{array}{cc}
\Lambda^{11}(q)+\frac{2}{g} & -\frac{i}{2} q_{\mu} \Lambda_{J 1}^{\mu} \\
-\frac{i}{2} q_{\mu} \Lambda_{1 J}^{\mu} & \frac{1}{4} q_{\mu} q_{\nu} \tilde{\Lambda}_{J J}^{\mu \nu}(q)
\end{array}\right) \zeta_{q}
$$

where $\zeta_{q} \equiv\left(|\Delta|_{q}, \theta_{q}\right)$. Here, we used the quadrivector notation $q^{\mu}=\left(i \Omega_{m}, \mathbf{q}\right), q_{\mu}=\left(i \Omega_{m},-\mathbf{q}\right)$, and we introduced the generalized current-current bubbles:

$$
\begin{gathered}
\tilde{\Lambda}_{J J}^{\mu \nu}=-\frac{n}{m} \eta^{\mu \nu}\left(1-\eta^{\nu 0}\right)+\Lambda_{J J}^{\mu \nu} \\
\Lambda_{J J}^{\mu \nu}=\frac{T}{V} \sum_{k} \operatorname{Tr}\left[\bar{G}_{k+q} \gamma^{\mu}(k, k+q) \bar{G}_{k} \gamma^{\nu}(k+q, k)\right] \\
\Lambda_{J i}^{\mu}=\frac{T}{V} \sum_{k} \operatorname{Tr}\left[\bar{G}_{k+q} \gamma^{\mu}(k, k+q) \bar{G}_{k} \sigma_{i}\right]
\end{gathered}
$$

where $\eta^{\mu \nu}=\operatorname{diag}(1,-1,-1)$ and the current vertex is

$$
\gamma^{\mu}(k, k+q)=\left(\sigma_{3}, \frac{\mathbf{k}+\mathbf{q} / 2}{m} \sigma_{0}\right) .
$$

Notice that the above definitions (A6), (A18), and (A19) are slightly redundant, since, e.g., $\Lambda_{J J}^{00} \equiv \Lambda_{33}$. Nevertheless, Eqs. (A18) and (A19) allow for a transparent interpretation of the phase mode in Eq. (A16), whose dispersion is given explicitly by

$$
q_{\mu} q_{v} \tilde{\Lambda}_{J J}^{\mu \nu}(q) \equiv-\Omega_{m}^{2} \tilde{\Lambda}_{J J}^{00}+q^{i} q^{j} \tilde{\Lambda}_{J J}^{i j}-2 i \Omega_{m} q^{i} \tilde{\Lambda}_{J J}^{0 i} .
$$

Indeed, since only time or spatial derivatives of the phase field enter the self-energy (A15), the hydrodynamic limit of the phase mode is easily obtained by taking the $q=0$ limit of the current-current fermionic bubbles, which appear as coefficients in Eq. (A21). More specifically, since $\Lambda_{J J}^{00}(0)=$ $-\kappa$, where $\kappa$ is the compressibility, $\Lambda_{J J}^{i j}(0)=\left(\rho_{s} / m\right) \delta_{i j}$ and $\Lambda_{J J}^{0 i}(0)=0$, one finds immediately that in the longwavelength limit the phase mode has the well-known soundlike dispersion

$$
q_{\mu} q_{\nu} \tilde{\Lambda}_{J J}^{\mu \nu}(q) \simeq-\omega^{2} \kappa+\mathbf{q}^{2} \frac{\rho_{s}}{m}
$$

which characterizes the Bogoliubov-Anderson mode. Instead, in the Cartesian notation of Eq. (A5), the dispersion of the phase mode must be obtained by performing the low- $q$ expansion of the $\Lambda_{22}(q)$ bubble. An alternative root is to exploit the equivalence between the two derivations (A5) and (A16), that must be valid at all orders in $q$. Here, we prove explicitly this equivalence by using the definitions of the fermionic bubbles and the identity: ${ }^{59}$

$$
\bar{G}_{k+q}^{-1} \sigma_{3}-\sigma_{3} \bar{G}_{k}^{-1}=q_{\mu} \gamma^{\mu}(k+q, k)-2 i \bar{\Delta} \sigma_{2} .
$$

One can then prove the three equalities:

$$
\begin{gathered}
q_{\mu} \Lambda_{J 2}^{\mu}(q)=2 i \bar{\Delta}\left[\Lambda_{22}(q)+\frac{2}{g}\right], \\
q_{\mu} \tilde{\Lambda}_{J J}^{\mu \nu}(q)=2 i \bar{\Delta} \Lambda_{2 J}^{v}(q), \\
q_{\mu} \tilde{\Lambda}_{J 1}^{\mu}=2 i \bar{\Delta} \Lambda_{21} .
\end{gathered}
$$

Let us show, for example, the demonstration of Eq. (A24). By means of the definitions (A6) and (A19) and the equivalence (A23), we have

$$
\begin{aligned}
q_{\mu} & \Lambda_{J 2}^{\mu}(q)-2 i \bar{\Delta} \Lambda_{22} \\
& =\frac{T}{V} \sum_{k} \operatorname{Tr}\left[\bar{G}_{k+q}\left(q_{\mu} \gamma^{\mu}(k, k+q)-2 i \bar{\Delta} \sigma_{2}\right) \bar{G}_{k} \sigma_{2}\right] \\
& =\frac{T}{V} \sum_{k} \operatorname{Tr}\left[\sigma_{3} \bar{G}_{k} \sigma_{2}\right]-\frac{T}{V} \sum_{k} \operatorname{Tr}\left[\bar{G}_{k+q} \sigma_{3} \sigma_{2}\right] \\
& =2 i \frac{T}{V} \sum_{k} \operatorname{Tr}\left[\bar{G}_{k} \sigma_{1}\right]=4 i \bar{\Delta} \Pi=\frac{4 i \bar{\Delta}}{g},
\end{aligned}
$$

where we used the definition of the $\Pi$ bubble, $\Pi \equiv$ $\frac{T}{2 V} \sum_{k} \operatorname{Tr}\left(\bar{G}_{k} \sigma_{1}\right)$ and the self-consistency equation (A4) $\Pi=$ $1 / g$. The remaining equalities (A25) and (A26) can be obtained with a similar procedure. ${ }^{59}$ By means of Eqs. (A24) and (A25) and using $\Lambda_{2 J}^{\mu}(q)=\Lambda_{J 2}^{\mu}(-q)=-\Lambda_{J 2}^{\mu}$, we then have

$$
\frac{1}{4} q_{\mu} q_{v} \tilde{\Lambda}_{J J}^{\mu v}(q)=-\frac{i}{2} \bar{\Delta} q_{\nu} \Lambda_{J 2}^{v}(q)=\bar{\Delta}^{2}\left[\Lambda_{22}(q)+\frac{2}{g}\right] \text {, }
$$

so that, since $\operatorname{Im} \Delta_{q}=\bar{\Delta} \theta_{q}$, we recover the equivalence between the phase-fluctuation propagator in Eqs. (A5) and (A16). Analogously, by means of Eq. (A26), we recover the equivalence in Eqs. (A5) and (A16) between the off-diagonal terms in the amplitude-phase fluctuations, completing the demonstration of the full equivalence of the two procedures. Notice that the above relations (A22) and (A28) allow one to easily derive the low-momentum expansion of the $\Lambda_{22}$ bubble, which has been used in Eq. (38) of the main text. Indeed, we have that

$$
\begin{aligned}
\Lambda^{22}(q) & =-2 \Pi+\frac{1}{4 \Delta^{2}} q_{\mu} q_{\nu} \tilde{\Lambda}_{J J}^{\mu \nu}(q) \\
& \simeq-2 \Pi+\frac{1}{4 \Delta^{2}}\left(\Omega_{m}^{2} \kappa+\mathbf{q}^{2} \frac{\rho_{s}}{m}\right) .
\end{aligned}
$$

Notice that in Eq. (A29) above the coefficient of the $\Omega_{m}^{2}$ term, i.e., the density-density correlation function $\Lambda_{J J}^{00}(q)$, has been taken in the static limit, where it gives the compressibility. On the other hand, as it is well known, ${ }^{9}$ at finite $T$, the dynamic limit of $\Lambda_{J J}^{00}(q)$, which is the one relevant to compute the collective modes at $\mathbf{q}=0$ in Secs. II and III, differs from $\kappa$. On the other hand, the low- $q$ expansion of the $\Lambda^{22}(q)$ can still be connected to the generalized current-current bubbles, which is the relation needed to recover the equivalence between the two derivations of the collective modes.

It is worth noting that in the single-band case, the description in terms of Cartesian or polar coordinates is equivalent since one usually chooses a gauge where the saddle-point gap value $\bar{\Delta}$ is real. Thus the two descriptions correspond 
to the same choice of fluctuations directions and are then trivially equivalent. On the other hand, if one had chosen a finite saddle-point phase for the gap, then the Cartesian description of fluctuations would be very inconvenient, since in this case, the $\Lambda_{12}(q=0)$ bubble, which couples real and imaginary parts in Eq. (A5), would be nonzero, as we already emphasized in Eq. (56) above for the TRSB. However, in the single-band case, one could still make a proper rotation to polar coordinates that would lead again to decoupled amplitude and phase fluctuations. The multiband TRSB case discussed in Sec. III B is instead different. Indeed, in the TRSB state, each gap acquires a nontrivial saddle-point value for the phase. In this situation, even thought the choice of polar coordinates for each band could still make the part of fluctuations described by fermionic bubbles orthogonal, the coupling matrix $\hat{g}_{l m}$ in Eq. (58) will not be diagonalized by this rotation, making amplitude and phase fluctuations always intrinsically mixed.

Finally, let us derive for the sake of completeness the relations between the average values of the HS field $\Delta_{q}$ and the physical correlation functions expressed in terms of the fermionic operators $\phi_{q}$ defined in Eq. (2) above. ${ }^{5}$ Let us discuss it for the case of Cartesian coordinates: starting from Eq. (A1), we add a source field $\Psi_{\mathbf{q}}$, which couples to $\phi_{q}$ such that

$$
\begin{aligned}
Z & =\int \mathcal{D} c_{\sigma} \mathcal{D} c_{\sigma}^{\dagger} \mathcal{D} \Delta \mathcal{D} \Delta^{\dagger} e^{-S+\sum_{q}\left(\phi_{q}^{\dagger} \Psi_{q}+\text { H.c. }\right)}, \\
\left\langle\phi_{q}\right\rangle & =\left.\frac{\partial \ln Z}{\partial \Psi_{q}^{*}}\right|_{\Psi=0}, \\
\left\langle\phi_{q}^{\dagger} \phi_{-q}\right\rangle & =\left.\frac{\partial^{2} \ln Z}{\partial \Psi_{q} \Psi_{-q}^{*}}\right|_{\Psi=0} .
\end{aligned}
$$

To perform explicitly the derivatives on the right-hand sides of Eqs. (A30) and (A31), we can notice that the total action in Eq. (A30) can be written as

$$
\begin{aligned}
S^{\prime}= & S-\sum_{q}\left(\phi_{q}^{\dagger} \Psi_{q}+\text { H.c. }\right) \\
= & \sum_{q} \frac{\left|\Delta_{q}\right|^{2}}{g}-\sum_{q}\left[\left(\Psi_{q}^{*}+\Delta_{q}^{*}\right) \phi_{q}+\text { H.c. }\right] \\
= & \sum_{q} \frac{\left|\tilde{\Delta}_{q}\right|^{2}}{g}-\sum_{q}\left[\tilde{\Delta}_{q}^{*} \phi_{q}+\text { H.c. }\right] \\
& +\sum_{q} \frac{\left|\Psi_{q}\right|^{2}}{g}-\frac{\left(\tilde{\Delta}_{q}^{*} \Psi_{q}+\text { H.c. }\right)}{g},
\end{aligned}
$$

where we shifted $\Psi_{q}+\Delta_{q}=\tilde{\Delta}_{q}$. One can then easily derive the relations

$$
\begin{aligned}
&\left.\frac{\partial \ln Z}{\partial \Psi_{q}^{*}}\right|_{\Psi=0}=\frac{1}{g}\left\langle\Delta_{q}\right\rangle=\left\langle\phi_{q}\right\rangle, \\
&\left.\frac{\partial^{2} \ln Z}{\partial \Psi_{q} \Psi_{-q}^{*}}\right|_{\Psi=0}=-\frac{1}{g}+\frac{1}{g^{2}}\left\langle\Delta_{q}^{*} \Delta_{-q}\right\rangle=\left\langle\phi_{q}^{\dagger} \phi_{-q}\right\rangle,
\end{aligned}
$$

where we also used the fact that at $\Psi=0$ the averages of the $\Delta_{q}$ and $\tilde{\Delta}_{q}$ fields coincide. By direct inspection on the Gaussian action (A5) for the HS-field fluctuations, we recover the well-known result that the correlator for the HS field $\Delta_{q}$ corresponds to the RPA resummation of the potential, while the correlator of the physical field gives the RPA resummation on the corresponding fermionic susceptibility. For example, for the real components, we have

$$
\begin{aligned}
\left\langle\operatorname{Re} \Delta_{-q} \operatorname{Re} \Delta_{q}\right\rangle & =\frac{g}{1+\frac{g}{2} \Lambda^{11}(q)}, \\
\left\langle\operatorname{Re} \phi_{-q} \operatorname{Re} \phi_{q}\right\rangle & =\frac{-\Lambda^{11}(q) / 2}{1+\frac{g}{2} \Lambda^{11}(q)} .
\end{aligned}
$$

\section{APPENDIX B: DERIVATION OF THE LEGGETT MODE FOR DOMINANT INTRABAND PAIRING}

In this Appendix, we show explicitly how the generalized transformation (9) can be used to obtain the Leggett mode in the two-band case with dominant intraband pairing, as it would be appropriate for example to $\mathrm{MgB}_{2} \cdot{ }^{20,21}$ As it has been shown in Ref. 20, in this case, one could easily obtain the Leggett's mode dispersion by using a straightforward generalization to a two-band case of the derivation (A16) reviewed above in terms of polar coordinates. By introducing a HS field for each band, with phases $\left(\theta_{1}(q), \theta_{2}(q)\right)$, the long-wavelength $\mathbf{q}=0$ phase fluctuations (decoupled from the amplitude ones) are described by the matrix

$$
\hat{S}_{\mathrm{FL}}^{P}=\frac{1}{8}\left(\begin{array}{cc}
-N_{1} \omega^{2}+A & -A \\
-A & -N_{2} \omega^{2}+A
\end{array}\right),
$$

where $A$ is a constant connected to the matrix $\hat{g}$ of the SC couplings and to the saddle-point gap values $\bar{\Delta}_{l}$ in each band:

$$
A=\frac{8 g_{12} \bar{\Delta}_{1} \bar{\Delta}_{2}}{\operatorname{det} \hat{g}} .
$$

In Eq. (B1), we recognize the $\omega^{2}$ expansion derived in Eq. (A21) above, with the compressibility $\kappa_{l}$ in each band approximated by the corresponding density of states $N_{l}$. The phase collective modes are found as usual as solutions of the equation $\operatorname{det} \hat{S}_{\mathrm{FL}}^{P}=0$. The first solution $\omega=0$ corresponds as to the Bogoliubov-Anderson (BA) mode, while a second solution exists corresponding to the Leggett mode: ${ }^{18}$

$$
\omega^{2}=\omega_{L}^{2}=A \frac{N_{1}+N_{2}}{N_{1} N_{2}} .
$$

Notice that, as already observed by Leggett in his original paper, ${ }^{18}$ this solution only exists when $A>0$, i.e., when $\operatorname{det} \hat{g}>0$ [since $\operatorname{sign}\left(\bar{\Delta}_{1} \bar{\Delta}_{2}\right)=\operatorname{sign} g_{12}$ ], which corresponds to intraband-dominated coupling. One can also easily verify that the BA mode corresponds to fluctuations having $\theta_{1}=\theta_{2}$, while the Leggett mode corresponds to

$$
\left(-N_{1} \omega_{L}^{2}+A\right) \theta_{1}-A \theta_{2}=0 \Rightarrow \frac{\theta_{1}}{\theta_{2}}=-\frac{N_{2}}{N_{1}},
$$

i.e., to antiphase oscillations in the two bands, weighted with the respective DOS. As usual, the Leggett mode (as well as the Carlson-Goldman one) can be equally found in linear-response theory. $^{21}$

Let us rewrite instead the effective phase-only action after using the generalized transformation $T$. In this case, since both the eigenvalues $\Lambda_{1}>\Lambda_{2}>0$ of $\hat{g}$ are positive, the hyperbolic matrix in Eq. (7) will be replaced by an ordinary rotation matrix, which preserves the structure $\operatorname{diag}(1,1)$ present in this 
case. The $T$ matrix will again be used to decouple the meanfield equations for the HS fields:

$$
\begin{aligned}
& \left(\begin{array}{cc}
\frac{1}{\Lambda_{1}}-\sum_{l} T_{1 l}^{2} \Pi_{l} & 0 \\
0 & \frac{1}{\Lambda_{2}}-\sum_{l} T_{2 l}^{2} \Pi_{l}
\end{array}\right)\left(\begin{array}{l}
\bar{h}_{1} \\
\bar{h}_{2}
\end{array}\right) \\
& \quad=Y_{1} \bar{h}_{1}+Y_{2} \bar{h}_{2}=0,
\end{aligned}
$$

where now no imaginary unit is associated to $\bar{h}_{2}$ since it decouples an ordinary bonding SC channel, and also $Y_{2}$ could in principle vanish. However, as soon as $Y_{1}=0$ and one enters the SC state, $Y_{2}$ can never vanish (see Appendix C), so the $\bar{h}_{1}$ will still be the only order parameter of the SC transition. The effective action will have the same structure of Eq. (36) derived above, with the remarkable difference that now the HF field $h_{2}$ used to decouple the $\Lambda_{2}$ channel will not carry out an additional $i$ unit, so that the $-1 / \Lambda_{2}$ term in Eq. (36) is replaced by $+1 / \Lambda_{2}$ :

$\hat{S}_{\mathrm{FL}}^{P}(q)=\left(\begin{array}{cc}\frac{1}{2} \sum_{l} \Lambda_{l}^{22}(q) T_{1 l}^{2}+\frac{1}{\Lambda_{1}} & \frac{1}{2} \sum_{l} \Lambda_{l}^{22} T_{1 l} T_{2 l} \\ \frac{1}{2} \sum_{l} \Lambda_{l}^{22} T_{1 l} T_{2 l} & \frac{1}{2} \sum_{l} \Lambda_{l}^{22}(q) T_{2 l}^{2}+\frac{1}{\Lambda_{2}}\end{array}\right)$.

To compute the above Eq. (B6) at $\mathbf{q}=0$ and small $\omega$, we use the expansion (38) of the $\Lambda_{22}$ bubbles, so that we obtain

$$
\hat{S}_{\mathrm{FL}}^{P}=-\left(\begin{array}{cc}
B \omega^{2} & C \omega^{2} \\
C \omega^{2} & D \omega^{2}-m^{2}
\end{array}\right),
$$

where

$$
\begin{gathered}
B=\frac{1}{8} \sum_{l} \frac{N_{l}}{\Delta_{l}^{2}} T_{1 l}^{2}, \\
D=\frac{1}{8} \sum_{l} \frac{N_{l}}{\Delta_{l}^{2}} T_{2 l}^{2}, \\
C=\frac{1}{8} \sum_{l} \frac{N_{l}}{\Delta_{l}^{2}} T_{1 l} T_{2 l}, \\
m^{2}=\frac{1}{\Lambda_{2}}-\sum_{l} \Pi_{l} T_{2 l}^{2} .
\end{gathered}
$$

Notice that $m^{2}$ in Eq. (B11) above is nothing else than the second eigenvalue $Y_{2}$ of the matrix of mean-field equations (B5) above, which is always nonzero below $T_{c}$ for a system with finite interband coupling. It is then clear that also the matrix (B7) leads to two solutions. The first one at $\omega=0$ corresponds to the BA mode: it involves only fluctuations of the $\operatorname{Im} h_{1, q}$ field, which thanks to the gap definitions (24) is indeed a uniform phase rotation for the gaps in both bands, see Eq. (40) above. The second solution is found at the frequency

$$
\omega^{2}=\frac{B m^{2}}{B D-C^{2}} .
$$

We will now show that Eq. (B12) coincides with the expression (B3) above, by deriving the explicit expressions of the $T$ matrix from the three conditions established in Sec. II: $\operatorname{det} T=1$, Eq. (22) $\sum_{l} T_{1 l} \Pi_{l} T_{2 l}=0$ and the saddle-point equation (23), which defines also the mean-field gaps $\Delta_{l}$ in Eq. (24). One can then easily show that

$$
T=\frac{1}{\sqrt{\sum_{l} \Pi_{l} \Delta_{l}^{2}}}\left(\begin{array}{cc}
\Delta_{1} / \sqrt{\Lambda_{1}} & \Delta_{2} / \sqrt{\Lambda_{1}} \\
-\sqrt{\Lambda_{1}} \Pi_{2} \Delta_{2} & \sqrt{\Lambda_{1}} \Pi_{1} \Delta_{1}
\end{array}\right),
$$

where we also have that the saddle-point value of the HS ordering field $\bar{h}_{1}$ is given by

$$
\bar{h}_{1}=\Lambda_{1} \sum_{l} \Pi_{l} \Delta_{l}^{2} .
$$

By means of Eqs. (B13) and (B14), we can then express the coefficents (B8)-(B11) in terms of the gap values $\Delta_{l}$ and of the eigenvalues, connected to the matrix $\hat{g}$ of the SC couplings. With lengthly but straightforward calculations, we then have

$$
\begin{gathered}
B=\frac{1}{8 \bar{h}_{1}^{2}} \sum_{l} N_{l}, \\
D=\frac{\Lambda_{1}^{2}}{8} \frac{N_{1} \Pi_{2}^{2} \Delta_{2}^{4}+N_{2} \Pi_{1}^{2} \Delta_{1}^{4}}{\Delta_{1}^{2} \Delta_{2}^{2}}, \\
C=\frac{\Lambda_{1}}{8 \bar{h}_{1}} \frac{-N_{1} \Pi_{2} \Delta_{2}^{2}+N_{2} \Pi_{1} \Delta_{1}^{2}}{\Delta_{1} \Delta_{2}}, \\
m^{2}=\frac{\Lambda_{1}}{\bar{h}_{1}}\left(1-\Pi_{1} \Pi_{2} \operatorname{det} \hat{g}\right)=\frac{g_{12}}{\operatorname{det} \hat{g} \Delta_{1} \Delta_{2}} .
\end{gathered}
$$

As a consequence, we get in Eq. (B12) that $\left(B D-C^{2}\right) / B=$ $N_{1} N_{2} / 8 \Delta_{1}^{2} \Delta_{2}^{2}\left(N_{1}+N_{2}\right)$, and we then recover the expression (B3) for the frequency of the second eigenmode. By means of the same relations, one can also prove that the eigenvector corresponding to the (B12) solution, i.e., $\operatorname{Im} h_{1}=$ $-(C / B) \operatorname{Im} h_{2}$ describes the antiphase fluctuations (B4) identified above for the Leggett mode.

\section{APPENDIX C: TRSB TRANSITION IN A THREE-BAND MODEL}

In this Appendix, we discuss the TRSB transition in the three-band case in terms of the action for the HS fields. In general, once given the matrix (47) of the SC couplings, we are interested to the case where there are two bonding eigenvalues $\Lambda_{1}$ and $\Lambda_{2}$ and one antibonding one $-\Lambda_{3}$. The $T=P_{\alpha, \beta, \varphi} R$ transformation in Eq. (9) is defined through the rotation $R$ which diagonalizes $g$ and the matrix $P_{\alpha, \beta, \varphi}$ consisting in a 3D Poincaré transformation in the $h$ space with $h_{1,2}$ being the spatial $(x, y)$ dimensions and $h_{3}$ the time $(t)$ one:

$$
\begin{aligned}
P_{\alpha, \beta, \varphi}= & \left(\begin{array}{ccc}
\frac{1}{\sqrt{\Lambda_{1}}} & 0 & 0 \\
0 & \frac{1}{\sqrt{\Lambda_{2}}} & 0 \\
0 & 0 & \frac{1}{\sqrt{\Lambda_{3}}}
\end{array}\right) R_{\alpha}^{x y} H_{\varphi} \vec{v}_{x y}(\beta), t \\
& \times\left(\begin{array}{ccc}
\sqrt{\Lambda_{1}} & 0 & 0 \\
0 & \sqrt{\Lambda_{2}} & 0 \\
0 & 0 & \sqrt{\Lambda_{3}}
\end{array}\right), \alpha, \beta \in[0,2 \pi], \varphi \in \mathbb{R} .
\end{aligned}
$$

Here, $R_{\alpha}^{x y}$ is an ordinary rotation of angle $\alpha$ (with respect to the $x$ axis) in the $(x, y)$ plane, while $H_{\varphi}^{\vec{v}_{x y}(\beta), t}$ is a hyperbolic rotation of angle $\varphi$ in the plane identified by the $t$ direction and by the versor $\vec{v}_{x y}(\beta)$ of the $(x, y)$ plane, $\beta$ being the angle with respect to $x$. After the HS decoupling, the equivalent of the action (12) will read

$$
\begin{aligned}
S= & S_{0}+\int d \tau d \mathbf{x} \frac{\left|h_{1}(x)\right|^{2}}{\Lambda_{1}}+\frac{\left|h_{2}(x)\right|^{2}}{\Lambda_{2}}+\frac{\left|h_{3}(x)\right|^{2}}{\left|\Lambda_{3}\right|} \\
& -\left(h_{1}^{*} \psi_{1}+h_{2}^{*} \psi_{2}+\text { H.c. }\right)-i\left(h_{3}^{*} \psi_{3}+\text { H.c. }\right) .
\end{aligned}
$$


We can then proceed as in the two-band case, having in mind that now the HS field $h_{3}$ associated to the antibonding channel will enter with an imaginary unit in both the saddle-point Green's function (14) and the self-energy (15), playing then the role of $h_{2}$ for the two-band case. We observe that the $T$ matrix depends on three parameters, i.e., the rotation angles $\alpha, \beta, \varphi$ of the matrix ( $\mathrm{C} 1)$ above: they are fixed (self-consistently) by the three conditions (48) above, which are used to decouple the saddle-point equations for the HS fields. In the ordinary SC phase, when only the $h_{1}$ field has a finite saddle-point value, the derivation of the action is a straightforward extension of the calculations presented in Sec. II.

Now, we prove briefly that, if $T_{\mathrm{TRSB}}$ exists such that Eq. (50) holds, then the system undergoes a second-order phase transition to a TRSB phase. In particular, $\bar{h}_{2}$ will emerge purely imaginary. First of all, we recall that the $T$ transformation is used to put the saddle-point equations (25) in diagonal form, see Eq. (26). In the ordinary TRS phase, only one element of the matrix $T \hat{\Pi} T^{T}-\hat{\Lambda}^{-1}$ vanishes, while at $T \leqslant T_{\mathrm{TRSB}}$, Eq. (50) holds and a second element vanishes. In this situation, the $h_{1}$ and $h_{2}$ spaces are degenerate and any additional rotation $\alpha$ in the transformation matrix $P_{\alpha, \beta, \varphi}$ will leave the result unchanged. Hence, if we define, in general, $\bar{h}_{i}=R_{i}+i I_{i}$, we can use the parameter $\alpha$, along with the U(1) gauge symmetry, to impose $I_{1}=R_{2}=0$. Indeed, even if at some $T<T_{\mathrm{TRSB}}$, one has $I_{1}, R_{2} \neq 0$, we can pass to an other solution $\left(\vec{R}^{\prime}, \vec{I}^{\prime}\right)$ with vanishing $I_{1}^{\prime}$ and $R_{2}^{\prime}$ by means of the transformation

$$
|\hat{\Lambda}|^{-1 / 2}\left(\begin{array}{cccc}
\cos \alpha & -\sin \alpha & 0 & 0 \\
\sin \alpha & \cos \alpha & 0 & 0 \\
0 & 0 & \cos \alpha & -\sin \alpha \\
0 & 0 & \sin \alpha & \cos \alpha
\end{array}\right)|\hat{\Lambda}|^{1 / 2}\left(\begin{array}{cccc}
\cos \vartheta & 0 & -\sin \vartheta & 0 \\
0 & \cos \vartheta & 0 & -\sin \vartheta \\
\sin \vartheta & 0 & \cos \vartheta & 0 \\
0 & \sin \vartheta & 0 & \cos \vartheta
\end{array}\right)\left(\begin{array}{c}
R_{1} \\
R_{2} \\
I_{1} \\
I_{2}
\end{array}\right)=\left(\begin{array}{c}
R_{1}^{\prime} \\
0 \\
0 \\
I_{2}^{\prime}
\end{array}\right)
$$

where $\vartheta$ is the $\mathrm{U}(1)$ angle. Once the possibility to choose $R_{2}=0$ is established, we should prove that at $T<T_{\mathrm{TRSB}}$, the ground state favors, indeed, a finite value of the imaginary part of $\bar{h}_{2}$. Indeed, even if at $T=T_{\mathrm{TRSB}}$, Eq. (50) is satisfied, at lower temperatures, there are still three possibilities: (i) $I_{2}$ remains zero and Eq. (50) does not hold anymore, so that $R_{1}$ remains the only order parameter; (ii) $\bar{h}_{2}$ opens with a real component $R_{2}$ only; or (iii) $\bar{h}_{2}$ opens with a finite imaginary component $I_{2}$, and a TRSB phase is established. To show that the case (iii) is the ground state, we make use of the fact that the imaginary fluctuations of $h_{2}$ at $q=0$ become massless at $T=T_{\mathrm{TRSB}}$, as proven in Eq. (52) above. Indeed, let us write down the expansion of the action at a temperature $T \lesssim T_{\mathrm{TRSB}}$ with respect to the mean-field action $\tilde{S}_{\mathrm{MF}}$ computed with the solution (i), i.e., $\vec{R}=\left(R_{1}, 0,0\right), \vec{I}=\overrightarrow{0}$. By using in the fluctuation action the $T$ matrix and the fermionic bubble evaluated at the expansion point and the results of Sec. III, we have

$$
\begin{aligned}
S= & \tilde{S}_{\mathrm{MF}}+\frac{1}{2} \delta \vec{R}^{T}\left(T \hat{\Lambda}^{11} T^{T}+2 \hat{\Lambda}^{-1}\right) \delta \vec{R} \\
& +\frac{1}{2} \delta \vec{I}^{T}\left(\hat{T} \hat{\Lambda}^{22} \hat{T}^{T}+2 \hat{\Lambda}^{-1}\right) \delta \vec{I}+\mathcal{O}\left(R^{3}, I^{3}, R I^{2}, I R^{3}\right),
\end{aligned}
$$

where $\delta \vec{R}, \delta \vec{I}$ are the displacements with respect to the expansion point and $\delta \vec{I}$ includes the imaginary factor $i$ of the antibonding channel, in accordance with the definition given above Eq. (52). Observe that in Eq. (C4), the linear terms do not appear since $S_{\mathrm{MF}}^{(1)}$ is a stationary point for the action, and the phase-amplitude couplings are absent since above $T_{\mathrm{TRSB}}$, we have chosen the gauge where all the gaps are real. Using the relations (37) and (38) for the $q=0$ values of the fermionic bubble, we can then see that $\delta \vec{R}$ fluctuations are always costly, so that case (ii) leads to an increase of the energy. On the other hand, thanks to the identity $\hat{\Lambda}_{22}(q=0)=-2 \hat{\Pi}$, the $\delta \vec{I}$ fluctuations have explicitly the form

$$
\begin{aligned}
& \delta \vec{I}^{T} \operatorname{diag}(0,-a,-b) \delta \vec{I} \\
& \quad=a^{\prime}\left(T-T_{\mathrm{TRSB}}\right) I_{2}^{2}+b I_{3}^{2}, \quad a, a^{\prime}, b>0,
\end{aligned}
$$

where we used the fact that at $T=T_{\mathrm{TRSB}}$ Eq. (50) holds and $\delta I_{2}$ fluctuations are massless. As one can see, $\delta I_{3}$ fluctuation increase the energy while $\delta I_{2}$ fluctuations decrease it, making the TRS phase unstable towards a phase with a finite $I_{2}$, which leads to nontrivial phases for the gaps (51) and then to a TRSB phase. This instability will be of course compensated by higher-order terms in the expansion (C4), that can also lead to a finite $\delta I_{1}$ and $\delta R_{2}$ along with a finite $\delta I_{2}$. However, as we discussed above, these components can be eliminated by the transformation (C3), making the definition (51) fully general.

We have then proven that the vanishing of a second eigenvalue of the matrix $\hat{\Pi}-\hat{g}^{-1}$, i.e., Eq. (50), is a sufficient condition for a TRSB phase, since when this happens the $\bar{h}_{2}$ field acquires a finite imaginary part. We will now show that this is also a necessary condition for having a TRSB phase. Let us go back to the set of self-consistency equations (25), and let us decompose the matrix $\hat{\Pi}-\hat{g}^{-1}$ in its eigenvectors at a temperature $T \leqslant T_{c}$ where the ordinary SC state is established:

$$
\hat{\Pi}-\hat{g}^{-1}=\lambda_{2}(T) \mathbf{u}_{2}^{T} \mathbf{u}_{2}+\lambda_{3}(T) \mathbf{u}_{3}^{T} \mathbf{u}_{3},
$$

where $\lambda_{i}(T)$ and $\mathbf{u}_{i}$ are real, since the matrix $\hat{\Pi}-\hat{g}^{-1}$ is real and symmetric. Here, we used the fact that below $T_{c}$ one eigenvalue vanishes, allowing for a finite solution $\vec{\Delta}$. In general, $\vec{\Delta}$ is a vector of complex numbers that satisfy the self-consistency equation (25), i.e.,

$$
\begin{aligned}
& \lambda_{2}(T)\left(\operatorname{Re} \vec{\Delta} \cdot \mathbf{u}_{2}\right) \mathbf{u}_{2}+\lambda_{3}(T)\left(\operatorname{Re} \vec{\Delta} \cdot \mathbf{u}_{3}\right) \mathbf{u}_{3}=0, \\
& \lambda_{2}(T)\left(\operatorname{Im} \vec{\Delta} \cdot \mathbf{u}_{2}\right) \mathbf{u}_{2}+\lambda_{3}(T)\left(\operatorname{Im} \vec{\Delta} \cdot \mathbf{u}_{3}\right) \mathbf{u}_{3}=0 .
\end{aligned}
$$

Since $\mathbf{u}_{2}$ and $\mathbf{u}_{3}$ are orthogonal, the above equations imply that the vectors $\operatorname{Re} \vec{\Delta}$ and $\operatorname{Im} \vec{\Delta}$ are also orthogonal to both $\mathbf{u}_{2}$ and $\mathbf{u}_{3}$. Thus either one of the two vanishes, or they are parallel to $\mathbf{u}_{1}$. In all these cases, the gaps have all the same phases, and then the state is TRS. On the other hand, when one additional eigenvalue vanishes in Eq. (C6), say $\lambda_{2}=0$, then $\operatorname{Re} \vec{\Delta}$ and $\operatorname{Im} \vec{\Delta}$ belong to the two-dimensional subspace spanned by $\mathbf{u}_{1}$ and $\mathbf{u}_{2}$, so that their phases can be complex. In this respect, 
it can also be instructive to show how simple geometrical arguments can be used to establish if a TRSB phase exists a $T=0$ for a generic coupling matrix. Let us start from Eq. (C6) with $\lambda_{2}=0$, and let us redefine $\mathbf{V}=\sqrt{\lambda_{3}} \mathbf{u}$, so that $\hat{\Pi}-\hat{g}^{-1}$ is written explicitly as a projector:

$$
\hat{\Pi}-\hat{g}^{-1}=\mathbf{V}^{T} \mathbf{V} \text {. }
$$

This equation allows one to determine $\mathbf{V}$ in terms only of the couplings: indeed, it gives explicitly

$$
g^{-1}=-\left(\begin{array}{ccc}
V_{1}^{2}-\Pi_{1} & V_{1} V_{2} & V_{1} V_{3} \\
V_{1} V_{2} & V_{2}^{2}-\Pi_{2} & V_{2} V_{3} \\
V_{3} V_{1} & V_{3} V_{2} & V_{3}^{2}-\Pi_{3}
\end{array}\right) .
$$

Thus temperature or band parameters, which only enter via the Cooper bubbles, do not affect the forbidden direction $\mathbf{V}$, which is fully determined as (let $\hat{G}$ be the inverse of $\hat{g}$ )

$$
\begin{aligned}
& V_{1}^{2}=-\frac{G_{12} G_{31}}{G_{23}}, \\
& V_{2}^{2}=-\frac{G_{21} G_{32}}{G_{13}}, \\
& V_{3}^{2}=-\frac{G_{31} G_{23}}{G_{12}} .
\end{aligned}
$$

Observe also that $V$ can be identically zero only if all the interband couplings vanish. Thus is any real multiband system, where at least one interband coupling is finite, there must exist one forbidden direction. In the two-band case, this guarantees that the second eigenvalue of the matrix $\hat{\pi}-\hat{g}^{-1}$ never vanishes. Once $\mathbf{V}$ is known, the self-consistency equations for the gap amplitudes are also determined by the diagonal terms in Eq. (C10), which give explicitly

$$
\Pi_{i}=G_{i i}+V_{i}^{2}=G_{i i}-\frac{G_{i k} G_{j i}}{G_{k j}} \quad(\forall i, k \neq j \neq i) .
$$

As one can see, in the TRSB phase, the equations for the gap amplitudes in the various bands decouple, and they all reduce to a single-band BCS equation with different effective couplings. This allows us also to express in the self-consistency equations (C7) and (C8) the gap amplitudes as a function of the Cooper bubbles, by inverting the relation $\Pi_{i}(T=0)=$ $N_{i} \operatorname{asinh}\left(\omega_{0} /|\Delta|_{i}\right)$. Thus Eqs. (C7) and (C8) above reduce in the TRSB state to

$$
\begin{aligned}
& \vec{V} \cdot \operatorname{Re} \vec{\Delta}=0 \Rightarrow \sum_{l} V_{l} \frac{\omega_{0} \cos \bar{\vartheta}_{l}}{\sinh \Pi_{l} / N_{0}^{l}}=0, \\
& \vec{V} \cdot \operatorname{Im} \vec{\Delta}=0 \Rightarrow \sum_{l} V_{l} \frac{\omega_{0} \sin \bar{\vartheta}_{l}}{\sinh \Pi_{l} / N_{0}^{l}}=0 .
\end{aligned}
$$

As usual, gauge invariance allows us to fix one of the phases to zero, say $\vartheta_{3}=0$. Thus Eqs. $(\mathrm{C} 15)$ and $(\mathrm{C} 16)$ determine the two remaining nontrivial phases $\vartheta_{1}$ and $\vartheta_{2}$ in terms only of the coupling matrix and band parameters, given by Eqs. (C11) (C14) above. For example, in the case of the coupling matrix considered in Eq. (45), one has for the inverse matrix (in units $N_{l}=N=1$ )

$$
G=-\frac{1}{2}\left(\begin{array}{ccc}
-\frac{1}{\eta} & \frac{1}{\eta} & \frac{1}{\lambda} \\
\frac{1}{\eta} & -\frac{1}{\eta} & \frac{1}{\lambda} \\
\frac{1}{\lambda} & \frac{1}{\lambda} & -\frac{\eta}{\lambda^{2}}
\end{array}\right),
$$

so that one immediately determines

$$
\begin{gathered}
V_{1}^{2}=V_{2}^{2}=\frac{1}{2 \eta}, \quad V_{3}^{2}=\frac{\eta}{2 \lambda^{2}}, \\
\Pi_{1}=\Pi_{2}=\frac{1}{\eta}, \quad \Pi_{3}=\frac{\eta}{\lambda^{2}} .
\end{gathered}
$$

Thus Eqs. (C15) and (C16) lead to $\vartheta_{1}=-\vartheta_{2}$, where

$$
\cos \theta_{1}=-\frac{\eta}{2 \lambda} \frac{\sinh \Pi_{1}}{\sinh \Pi_{3}} \simeq-\frac{\eta}{2 \lambda} \exp \left(\frac{1}{\eta}-\frac{\eta}{\lambda^{2}}\right),
$$

which admits, at fixed $\eta$, a finite solution only for $\lambda \leqslant \lambda_{\text {cr }}(\eta)$, see the phase diagram shown in Fig. 3.
${ }^{1}$ I. Mazin, Nature (London) 464, 183 (2010).

${ }^{2}$ D. N. Basov and A. V. Chubukov, Nat. Phys. 7, 272 (2011).

${ }^{3}$ P. J. Hirschfeld, M. M. Korshunov, and I. I. Mazin, Rep. Prog. Phys.

74, 124508 (2011).

${ }^{4}$ X. X. Xi, Rep. Prog. Phys. 71, 116501 (2008).

${ }^{5}$ S. De Palo, C. Castellani, C. Di Castro, and B. K. Chakraverty, Phys. Rev. B 60, 564 (1999).

${ }^{6}$ A. Paramekanti, M. Randeria, T. V. Ramakrishnan, and S. S. Mandal, Phys. Rev. B 62, 6786 (2000).

${ }^{7}$ L. Benfatto, S. Caprara, C. Castellani, A. Paramekanti, and M. Randeria, Phys. Rev. B 63, 174513 (2001).

${ }^{8}$ H. J. Kwon, A. T. Dorsey, and P. J. Hirschfeld, Phys. Rev. Lett. 86, 3875 (2001).

${ }^{9}$ S. G. Sharapov, H. Beck, and V. M. Loktev, Phys. Rev. B 64, 134519 (2001).

${ }^{10}$ S. G. Sharapov and H. Beck, Phys. Rev. B 65, 134516 (2002).

${ }^{11}$ L. Benfatto, A. Toschi, and S. Caprara, Phys. Rev. B 69, 184510 (2004).
${ }^{12}$ F. J. Burnell, Jiangping Hu, Meera M. Parish, and B. A. Bernevig, Phys. Rev. B 82, 144506 (2010).

${ }^{13}$ Y. Ota, M. Machida, T. Koyama, and H. Aoki, Phys. Rev. B 83, 060507(R) (2011).

${ }^{14}$ J. Carlström, J. Garaud, and E. Babaev, Phys. Rev. B 84, 134518 (2011).

${ }^{15}$ S. Z. Lin and X. Hu, Phys. Rev. Lett. 108, 177005 (2012).

${ }^{16}$ V. Stanev, Phys. Rev. B 85, 174520 (2012).

${ }^{17}$ S. Maiti and A. V. Chubukov, Phys. Rev. B 87, 144511 (2013).

${ }^{18}$ A. J. Leggett, Prog. Theor. Phys. 36, 901 (1966).

${ }^{19}$ M. V. Klein and S. B. Dierker, Phys. Rev. B 29, 4976 (1984).

${ }^{20}$ S. G. Sharapov, V. P. Gusynin, and H. Beck, Eur. Phys. J. B 30, 45 (2002).

${ }^{21}$ A. Anishchanka, A. F. Volkov, and K. B. Efetov, Phys. Rev. B 76, 104504 (2007)

${ }^{22}$ G. Blumberg, A. Mialitsin, B. S. Dennis, M. V. Klein, N. D. Zhigadlo, and J. Karpinski, Phys. Rev. Lett. 99, 227002 (2007).

${ }^{23}$ M. V. Klein, Phys. Rev. B 82, 014507 (2010). 
${ }^{24}$ V. Stanev and Z. Tesanovic, Phys. Rev. B 81, 134522 (2010).

${ }^{25}$ R. Thomale, C. Platt, W. Hanke, J. Hu, and B. A. Bernevig, Phys. Rev. Lett. 107, 117001 (2011).

${ }^{26}$ K. Suzuki, H. Usui, and K. Kuroki, Phys. Rev. B 84, 144514 (2011).

${ }^{27}$ S. Maiti, M. M. Korshunov, and A. V. Chubukov, Phys. Rev. B 85, 014511 (2012).

${ }^{28}$ See, e.g., Ref. 11 for a detailed description of the single-band case, and further details in Appendix A.

${ }^{29}$ L. Fanfarillo, L. Benfatto, S. Caprara, C. Castellani, and M. Grilli, Phys. Rev. B 79, 172508 (2009).

${ }^{30}$ R. M. Fernandes and A. J. Millis, Phys. Rev. Lett. 111, 127001 (2013).

${ }^{31}$ L. Benfatto, M. Capone, S. Caprara, C. Castellani, and C. Di Castro, Phys. Rev. B 78, 140502(R) (2008).

${ }^{32}$ L. Benfatto, E. Cappelluti, and C. Castellani, Phys. Rev. B 80, 214522 (2009).

${ }^{33}$ J. Hubbard, Phys. Rev. Lett. 3, 77 (1959).

${ }^{34}$ Notice that the $T$ matrix is not unitary, so the two combinations of fields $\psi_{1}$ and $\psi_{2}$ are not orthogonal. However, this is not a problem since only the $\psi_{1}$ combination of fermionic field will turn out to be the relevant $\mathrm{SC}$ channel to describe the mean-field solution.

${ }^{35}$ A. V. Vagov, A. A. Shanenko, M. V. Milosević, V. M. Axt, and F. M. Peeters, Phys. Rev. B 85, 014502 (2012).

${ }^{36}$ N. V. Orlova, A. A. Shanenko, M. V. Milosević, F. M. Peeters, A. V. Vagov, and V. M. Axt, Phys. Rev. B 87, 134510 (2013).

${ }^{37}$ A. F. Volkov and S. M. Kogan, Zh. Eksp. Teor. Fiz. 65, 2039 (1973) [Sov. Phys. JETP 38, 1018 (1974)].

${ }^{38}$ A. Schmid and G. Schoen, Phys. Rev. Lett. 34, 941 (1975).

${ }^{39}$ I. O. Kulik et al., J. Low. Temp. Phys. 43, 591 (1981).

${ }^{40}$ S. Graser, P. J. Hirschfeld, T. Maier, and D. J. Scalapino, New J. Phys. 11, 025016 (2009).

${ }^{41}$ S. Maiti, M. M. Korshunov, T. A. Maier, P. J. Hirschfeld, and A. V. Chubukov, Phys. Rev. Lett. 107, 147002 (2011); Phys. Rev. B 84, 224505 (2011).

${ }^{42}$ K. Nakayama, T. Sato, P. Richard, Y.-M. Xu, T. Kawahara, K. Umezawa, T. Qian, M. Neupane, G. F. Chen, H. Ding, and T. Takahashi, Phys. Rev. B 83, 020501(R) (2011).

${ }^{43}$ W. Malaeb, T. Shimojima, Y. Ishida, K. Okazaki, Y. Ota, K. Ohgushi, K. Kihou, T. Saito, C. H. Lee, S. Ishida, M. Nakajima, S. Uchida, H. Fukazawa, Y. Kohori, A. Iyo, H. Eisaki, C.-T. Chen, S. Watanabe, H. Ikeda, and S. Shin, Phys. Rev. B 86, 165117 (2012).
${ }^{44}$ D. Watanabe, T. Yamashita, Y. Kawamoto, S. Kurata, Y. Mizukami, T. Ohta, S. Kasahara, M. Yamashita, T. Saito, H. Fukazawa, Y. Kohori, S. Ishida, K. Kihou, C. H. Lee, A. Iyo, H. Eisaki, A. B. Vorontsov, T. Shibauchi, and Y. Matsuda, arXiv:1307.3408.

${ }^{45}$ K. Hashimoto et al., Phys. Rev. B 82, 014526 (2010).

${ }^{46}$ J.-Ph. Reid, M. A. Tanatar, A. Juneau-Fecteau, R. T. Gordon, S. Rene de Cotret, N. Doiron-Leyraud, T. Saito, H. Fukazawa, Y. Kohori, K. Kihou, C. H. Lee, A. Iyo, H. Eisaki, R. Prozorov, and Louis Taillefer, Phys. Rev. Lett. 109, 087001 (2012).

${ }^{47}$ F. F. Tafti, A. Juneau-Fecteau, M-È. Delage, S. René de Cotret, J-Ph. Reid, A. F. Wang, X-G. Luo, X. H. Chen, N. Doiron-Leyraud, and Louis Taillefer, Nat. Phys. 9, 349 (2013).

${ }^{48}$ K. Okazaki, Y. Ota, Y. Kotani, W. Malaeb, Y. Ishida, T. Shimojima, T. Kiss, S. Watanabe, C.-T. Chen, K. Kihou, C. H. Lee, A. Iyo, H. Eisaki, T. Saito, H. Fukazawa, Y. Kohori, K. Hashimoto, T. Shibauchi, Y. Matsuda, H. Ikeda, H. Miyahara, R. Arita, A. Chainani, and S. Shin, Science 337, 1314 (2012).

${ }^{49}$ C. Platt, R. Thomale, C. Honerkamp, S.-C. Zhang, and W. Hanke, Phys. Rev. B 85, 180502(R) (2012).

${ }^{50}$ R. M. Fernandes and A. J. Millis, Phys. Rev. Lett. 110, 117004 (2013).

${ }^{51}$ W.-C. Lee, S.-C. Zhang, and C. Wu, Phys. Rev. Lett. 102, 217002 (2009).

${ }^{52}$ R. Nandkishore, L. Levitov, and A. Chubukov, Nat. Phys. 8, 158 (2012).

${ }^{53}$ M. L. Kiesel, C. Platt, W. Hanke, D. A. Abanin, and R. Thomale, Phys. Rev. B 86, 020507 (2012).

${ }^{54}$ Wan-Sheng Wang, Yuan-Yuan Xiang, Qiang-Hua Wang, Fa Wang, Fan Yang, and Dung-Hai Lee, Phys. Rev. B 85, 035414 (2012).

${ }^{55}$ M. Kiesel, C. Platt, W. Hanke, and R. Thomale, Phys. Rev. Lett. 111, 097001 (2013).

${ }^{56}$ J. Goryo, M. H. Fischer, and M. Sigrist, Phys. Rev. B 86, 100507 (2012).

${ }^{57}$ P. K. Biswas, H. Luetkens, T. Neupert, T. Stuerzer, C. Baines, G. Pascua, A. P. Schnyder, M. H. Fischer, J. Goryo, M. R. Lees, H. Maeter, F. Bruckner, H.-H. Klauss, M. Nicklas, P. J. Baker, A. D. Hillier, M. Sigrist, A. Amato, and D. Johrendt, Phys. Rev. B 87, 180503 (2013).

${ }^{58}$ P. L. Carlson and A. M. Goldman, Phys. Rev. Lett. 34, 11 (1975).

${ }^{59}$ H. Guo, C.-C. Chien, and Y. He, J. Low Temp. Phys. 172, 5 (2013). 